\title{
Modelling photoluminescence from small particles
}

\section{General formalism and a simple reference implementation ${ }^{\star}$}

\author{
G. Malloci ${ }^{1,2,4}$, G. Mulas ${ }^{1,4}$, and P. Benvenuti ${ }^{2,3}$ \\ 1 INAF - Osservatorio Astronomico di Cagliari - AstroChemistry Group, Strada n.54, Loc. Poggio dei Pini, \\ 09012 Capoterra (CA), Italy \\ e-mail: gmulas@ca.astro.it \\ 2 Dipartimento di Fisica, Università degli Studi di Cagliari, S.P. Monserrato-Sestu Km 0.7, 09042 Cagliari, Italy \\ 3 INAF, Viale del Parco Mellini 84, 00136 Roma, Italy \\ e-mail: benvenuti@inaf.it \\ 4 Astrochemical Research in Space Network \\ http://www. ars-network.org
}

Received 17 November 2003 / Accepted 23 February 2004

\begin{abstract}
We developed a general recipe able to extrapolate the expected photoluminescence of small particles starting from available laboratory results obtained on bulk samples. We present numerical results for the simplest case, namely a spherical, homogeneous dust particle, in the limit of strongly localised fluorescence. In particular, our theoretical derivation produces an explicit, analytical, dependence of the photoluminescence spectrum on the angle between the direction of observation and that of the incoming exciting light. We expect the present model to be a useful tool that will allow the study of photoluminescence phenomena of interstellar dust to move beyond the straight, plain, possibly misleading comparison with experimental data on bulk samples.
\end{abstract}

Key words. ISM: dust, extinction - ISM: lines and bands - methods: numerical - radiation mechanisms: general radiative transfer - scattering

\section{Introduction}

Interstellar dust is thought to be a diluted dispersion of sub $\mu$ m-sized solid grains, probably with a complex, multilayered, and fluffy structure (see e.g., Mathis \& Whiffen 1989; Li \& Greenberg 1997). Regardless of their actual shape, such small particles are bound to have optical properties that are very different from those of the bulk material they are made of, the difference being about as large as that between the respective extinction properties, just for the same reasons. Therefore, any attempts to quantitatively compare photoluminescence (PL) signals from interstellar particles with experimental data obtained on bulk samples must carefully take into account the effect of particle size and optical properties. In particular, PL will undergo self-absorption and scattering within the dust particle before leaving it, and unless one is considering dust grains that are utterly non-absorbing at the wavelength of the PL, this effect is far from negligible.

\footnotetext{
Send offprint requests to: G. Malloci,

e-mail: gmalloci@ca.astro.it

* Appendix A is only available in electronic form at http://www.edpsciences.org
}

Hence, for meaningful quantitative results, one ought to compare astronomical interstellar PL phenomena, such as the Extended Red Emission (see e.g., Duley 2001; Smith \& Witt 2002), with laboratory data taken on samples as similar as possible to interstellar dust. If this is not feasible, one should use a detailed PL model to bridge the gap between experimental bulk properties and small particles, very much in the same way as the extinction properties of dust are usually computed from the knowledge of the complex refractive index of the material they are made of (see e.g., Bohren \& Huffman 1998). We present here such a model, in which we represent the emission as stemming from a density of uncorrelated oscillating electric dipoles, whose distribution is a functional of the locally absorbed energy. We expect this representation to be valid as long as the particle being modelled can be described in terms of classical electromagnetic theory (i.e. quantum effects are not taken into consideration at all). The details of our theoretical approach and its limits of applicability are described in Sect. 2. As a quantitative test, we show its specific implementation for a homogeneous, isotropic sphere in Sect. 3, where we present numerical results. For a realistic "proof of concept" test, we used the optical properties of processed organic refractory residues which are thought to be abundant in the diffuse 
interstellar medium. Section 5 draws the main conclusions of the present, germinal work, and outlines the directions of its foreseen development. Finally, formal derivation details are reported in Appendix A.

\section{The model}

The starting point of our model is the assumption that the photoluminescence (PL) power emitted by a given volume element $\mathrm{d} V$ is a functional of the electromagnetic energy absorbed in a neighbourhood around it.

As a first step, we thus need to be able to thoroughly solve the problem of the scattering and absorption of an electromagnetic wave hitting the dust particle under consideration. In particular we need to know the divergence of the Poynting vector $\boldsymbol{S}(\omega)$ associated to the Fourier component of angular frequency $\omega$ of the electromagnetic fields $\left(\boldsymbol{E}_{1}(\omega), \boldsymbol{H}_{1}(\omega)\right)$ within the particle. This quantity is commonly referred to as the source function $\mathcal{E}(\boldsymbol{r}, \omega)$ in classical electromagnetic theory (Dusel et al. 1976). $\mathcal{E}(\boldsymbol{r}, \omega) \mathrm{d} V \mathrm{~d} \omega$ is the power absorbed by the unit volume $\mathrm{d} V$ at position $\boldsymbol{r}$ from the Fourier component of the fields of angular frequency between $\omega$ and $\omega+\mathrm{d} \omega$. From the knowledge of $\mathcal{E}(\boldsymbol{r}, \omega)$ we can define the photon absorption rate per unit angular frequency and unit volume as:

$\mathcal{N}(\boldsymbol{r}, \omega)=\frac{\mathcal{E}(\boldsymbol{r}, \omega)}{\hbar \omega}$

Every absorbed photon of energy higher than a given threshold will give rise to one or more PL photons. We now define $p\left(\omega, \omega^{\prime}, \boldsymbol{r}, \boldsymbol{r}^{\prime}\right) \mathrm{d} \omega^{\prime} \mathrm{d} V^{\prime}$ as the average number of PL photons of frequency between $\omega^{\prime}$ and $\omega^{\prime}+\mathrm{d} \omega^{\prime}$ emitted by a volume element $\mathrm{d} V^{\prime}$ centred around the position $\boldsymbol{r}^{\prime}$ in the grain, following the absorption of a photon of frequency $\omega$ at the position $r$. To some extent, this may be thought of as a probability, but this naïve interpretation breaks down in the case where one absorbed photon may give rise to the emission of several PL photons. This representation clearly assumes absorption-emission sequences to be independent, i.e. the material is supposed to be able to completely relax after each absorption, thus nonlinear effects are completely neglected. This is obviously appropriate for solid state PL in interstellar environments.

In general, $p$ will vanish for large distances between $r$ and $\boldsymbol{r}^{\prime}$; the actual size $s$ of the neighbourhood of $\boldsymbol{r}$ which contributes to PL coming from it will depend on the material. The specific behaviour of $p$ in $\boldsymbol{r}$ will thus depend both on the material and on the shape of the sample in this whole neighbourhood. If $s$ is small with respect to the size of the sample, and if the sample is large enough to have "bulk" optical properties, boundary effects can be neglected in all but a thin "skin" close to the surface, and $p$ will only depend on the difference $\boldsymbol{r}-\boldsymbol{r}^{\prime}$; if the material is furthermore isotropic (which we will assume), $p$ will only depend on $\left\|\boldsymbol{r}-\boldsymbol{r}^{\prime}\right\|$. We remark that theoretical computations of the density of states in clusters of atoms yield results virtually undistinguishable from the bulk for atom numbers as small as a few hundreds, the specific value depending on the material considered (see e.g., Jena et al. 1992). This means that the depth of the "skin" with optical properties significantly different from the bulk will typically be a few molecular layers, i.e. of the order of a nanometer. Therefore, unless PL is dominated by the surface, it is generally safe to assume bulk optical properties for particles significantly larger than $\sim 10 \mathrm{~nm}$.

The integral of $p$ over $\omega^{\prime}$ and $V^{\prime}$ will yield the overall efficiency of PL following the absorption of a photon of a given energy in a given part of the dust grain. This cannot exceed unity, unless multiple photons can be emitted upon the absorption of one. In real life situations, this yield is usually much lower than unity, since even exceedingly efficient fluorescent materials usually show efficiencies of the order of $\sim 20 \%$ at most. The detailed variation of $p$ on the distance $\boldsymbol{r}-\boldsymbol{r}^{\prime}$ will depend on the properties of the material, but it becomes very simple in the following two extreme cases:

1. if $s$ is much smaller than the characteristic sizes of the sample considered and the wavelengths involved, the excitation of PL is effectively local, i.e. $p\left(\omega, \omega^{\prime}, \boldsymbol{r}, \boldsymbol{r}^{\prime}\right)$ may be considered to be proportional to a Dirac delta with respect to $\left\|\boldsymbol{r}-\boldsymbol{r}^{\prime}\right\|$; the condition for this to be a good approximation can be quantitatively expressed as $\left|k_{1} s\right| \ll 1, k_{1}=\omega N_{1} / c$ being the propagation constant in the material considered, whose complex refractive index is $N_{1}$;

2. on the other extreme, if $s$ is much larger than the size of the sample, any spatial correlation between absorption and emission is lost, and we will define the resulting PL as "pseudo-thermal", for reasons that will be made clear later.

The above limiting cases, both of which make the problem tractable, may be actually applicable to real physical situations: Hydrogenated Amorphous Carbon (HAC), as treated by Robertson (1996) and Seahar \& Duley (1999), fits neatly in the first, since the excitation energy of an absorbed photon is thought to be confined in the graphitic platelet in which it was absorbed, the same graphitic platelet then emitting the resulting PL photon in a very short time, of the order of $10^{-8} \mathrm{~s}$; bandgap PL by a homogeneous semiconductor, instead, may be an example of the second limiting case, as the absorption of a photon creates an electron-hole pair whose mean free path may easily be larger than the dimension of even a not too small dust grain.

We emphasise that we cite the two cases above merely as possible examples of the practical applicability of our approach, which is by no means limited to them. The present approach would not fail even if one or both of the cited examples should fail to fulfil the requirements for its applicability, which are indeed clearly and quantitatively stated. It would be proven to be inapplicable only if an example should be found which indeed fulfils the stated limits of applicability and shows a behaviour in contrast with our calculations. We will here restrict ourselves to these two limiting cases.

\subsection{The pseudo-thermal case}

The simplest case is that of "pseudo-thermal" PL. In this case, $p=p_{\mathrm{pt}}$ does not depend on $\boldsymbol{r}-\boldsymbol{r}^{\prime}$ at all, but only on $\omega, \omega^{\prime}$ and on the material and geometry of the sample. Multiplying $p$ by $\mathcal{N}(\omega, \boldsymbol{r})$ as defined in Eq. (1) and integrating over all $\omega$ and over the volume $\mathcal{V}$ of the particle yields the rate $\frac{\mathrm{d} f_{\mathrm{pt}}}{\mathrm{d} \omega^{\prime} \mathrm{d} V^{\prime}}$ of 
emission of PL photons per unit emitting volume $\mathrm{d} V^{\prime}$ and unit frequency interval $\mathrm{d} \omega^{\prime}$, produced by absorption over the whole particle at all frequencies:

$\frac{\mathrm{d} f_{\mathrm{pt}}}{\mathrm{d} \omega^{\prime} \mathrm{d} V^{\prime}}=\int \mathrm{d} \omega \int_{\mathcal{V}} \mathrm{d} V \mathcal{N}(\omega, \boldsymbol{r}) p_{\mathrm{pt}}\left(\omega, \omega^{\prime}\right)$.

Since $p_{\mathrm{pt}}$ does not depend on $\boldsymbol{r}$, the integral over $\mathcal{V}$ only operates on $\mathcal{N}(\omega, \boldsymbol{r})$ to yield the total number $\mathcal{N}_{\text {tot }}(\omega)$ of photons of frequency between $\omega$ and $\omega+\mathrm{d} \omega$ absorbed by the whole grain, to obtain

$\frac{\mathrm{d} f_{\mathrm{pt}}}{\mathrm{d} \omega^{\prime} \mathrm{d} V^{\prime}}=\int \mathrm{d} \omega \mathcal{N}_{\mathrm{tot}}(\omega) p_{\mathrm{pt}}\left(\omega, \omega^{\prime}\right)$

In turn, $\frac{\mathrm{d} f_{\mathrm{pt}}}{\mathrm{d} \omega^{\prime} \mathrm{d} V^{\prime}}$ depends neither on $\boldsymbol{r}$ nor on $\boldsymbol{r}^{\prime}$, and is obviously independent of polarisation. These properties are also enjoyed by the rate $\frac{\mathrm{d} f_{\mathrm{th}}}{\mathrm{d} \omega^{\prime} \mathrm{d} V^{\prime}}$ of thermal emission of photons per unit volume and unit frequency interval. In thermal emission, $\frac{\mathrm{d} f_{\mathrm{th}}}{\mathrm{d} \omega^{\prime} \mathrm{d} V^{\prime}}$ can be derived from the principle of detailed balance, and must equal the number of thermal background photons of that same frequency interval absorbed per unit time by the same unit volume of the dust particle. This, in turn, equals the flux $\Phi_{\text {int }}\left(\omega^{\prime}\right)$ of blackbody photons ${ }^{1}$ inside the dust grain times the absorption coefficient $\alpha\left(\omega^{\prime}\right)$ of the material composing it, times the whole solid angle $4 \pi$, namely

$\frac{\mathrm{d} f_{\mathrm{th}}}{\mathrm{d} \omega^{\prime} \mathrm{d} V^{\prime}}=4 \pi \alpha\left(\omega^{\prime}\right) \Phi_{\text {int }}\left(\omega^{\prime}\right)$

where

$\alpha\left(\omega^{\prime}\right)=\frac{2 \omega^{\prime} \kappa\left(\omega^{\prime}\right)}{c}$

and $\kappa\left(\omega^{\prime}\right)$ is the imaginary part of the refractive index of the dust grain $N_{1}$, which is frequency-dependent. Inverting Eq. (4) we obtain:

$\Phi_{\text {int }}\left(\omega^{\prime}\right)=\frac{1}{4 \pi \alpha\left(\omega^{\prime}\right)} \frac{\mathrm{d} f_{\text {th }}}{\mathrm{d} \omega^{\prime} \mathrm{d} V^{\prime}}$.

$\Phi_{\text {int }}\left(\omega^{\prime}\right)$ is proportional to the Planck function $B\left(\omega^{\prime}\right)$ and is given by:

$\Phi_{\text {int }}\left(\omega^{\prime}\right)=\frac{\left\{\mathfrak{R}\left[m_{1}\left(\omega^{\prime}\right)\right]\right\}^{2} B\left(\omega^{\prime}\right)}{\hbar \omega^{\prime}}$,

where $m_{1}=N_{1} / N$ is the relative refractive index and $\mathfrak{R}$ denotes the real part of its argument. The resulting overall rate per unit frequency interval of emission of thermal photons from the particle is given by the Kirchhoff law:

$F_{\text {th }}=4 \pi C_{\text {abs }}\left(\omega^{\prime}\right) \frac{B\left(\omega^{\prime}\right)}{\hbar \omega^{\prime}}$,

where $C_{\text {abs }}\left(\omega^{\prime}\right)$ is the absorption cross-section of the grain for incident light of frequency $\omega^{\prime}$. This overall emission is the result of the uniform thermal emission inside the dust grain,

\footnotetext{
${ }^{1}$ Expressed as the number of photons per unit frequency interval, confined to a unit solid angle about any direction, crossing a unit area normal to this direction in unit time.
}

attenuated by self-absorption. Using the above equations we obtain

$F_{\text {th }}=4 \pi C_{\text {abs }}\left(\omega^{\prime}\right) \frac{\Phi_{\text {int }}\left(\omega^{\prime}\right)}{\left\{\Re\left[m_{1}\left(\omega^{\prime}\right)\right]\right\}^{2}}$,

and thus

$F_{\text {th }}=\frac{C_{\text {abs }}\left(\omega^{\prime}\right)}{\alpha\left(\omega^{\prime}\right)\left\{\mathfrak{R}\left[m_{1}\left(\omega^{\prime}\right)\right]\right\}^{2}} \frac{\mathrm{d} f_{\mathrm{th}}}{\mathrm{d} \omega^{\prime} \mathrm{d} V^{\prime}}$.

The effect of self-absorption is therefore completely contained in the factor multiplying $\frac{\mathrm{d} f_{\text {th }}}{\mathrm{d} \omega^{\prime} \mathrm{d} V^{\prime}}$. Equation (7) relates the probability of emission of a single photon of a given frequency from a given position in the grain to the probability it has to emerge, under the hypotheses that the probability of emission is uniform inside the grain and that the optical properties of the grain are known. Since $\frac{\mathrm{d} f_{\mathrm{pt}}}{\mathrm{d} \omega^{\prime} \mathrm{d} V^{\prime}}$ and $\frac{\mathrm{d} f_{\mathrm{th}}}{\mathrm{d} \omega^{\prime} \mathrm{d} V^{\prime}}$ have exactly the same dependence on the position inside the dust grain, namely no dependence, the effect of self-absorption must be exactly the same, provided that the optical properties do not change, giving for the overall PL in this case:

$F_{\mathrm{pt}}=\frac{C_{\mathrm{abs}}\left(\omega^{\prime}\right)}{\alpha\left(\omega^{\prime}\right)\left\{\mathfrak{R}\left[m_{1}\left(\omega^{\prime}\right)\right]\right\}^{2}} \frac{\mathrm{d} f_{\mathrm{pt}}}{\mathrm{d} \omega^{\prime} \mathrm{d} V^{\prime}}$,

hence the name "pseudo-thermal" with which we labelled this case. The crucial step in the derivation above is that $p_{\mathrm{pt}}$ is constant inside the particle, which is only reasonable if the dust grain is considered homogeneous. In particular, the assumption of homogeneity implies that the complex refractive index is assumed to be constant and equal to its bulk value, which in turn means neglecting boundary effects. The effect of selfabsorption, in this case, is given mainly by two parts, which can be very simply interpreted from a physical point of view: the $\alpha\left(\omega^{\prime}\right)$ factor in the denominator says that if the material is strongly absorbing, only a sheet below the surface of thickness of the order of a few times $1 / \alpha\left(\omega^{\prime}\right)$ will contribute to PL; the other terms, particularly the $C_{\text {abs }}\left(\omega^{\prime}\right)$ term, stem from the effect that size and shape of the dust grain have on its optical properties, in a manner exactly analogous to the way they affect extinction.

Equation (8) is of limited practical use, since $\frac{\mathrm{d} f_{\mathrm{pt}}}{\mathrm{d} \omega^{\prime} \mathrm{d} V^{\prime}}$ itself can have, in principle, a nontrivial dependence on particle size and shape, which can only be obtained by direct measurements on appropriately sized and shaped samples. It is elegant in that it does show explicitly that size and shape effects can be quite relevant in this limiting case of pseudo-thermal PL and thus measures on bulk laboratory samples are bound to be useless for direct comparison with astronomical PL stemming from small particles.

\subsection{The local case}

The other extreme case which, although much more complicated, is still tractable, is the "local" one, in which each PL photon is emitted from the same position in the dust grain in which the corresponding exciting photon was absorbed. In this case, 
due to the extreme confinement of the excitation energy, we assume:

$p\left(\omega, \omega^{\prime}, \boldsymbol{r}, \boldsymbol{r}^{\prime}\right)=p_{\mathrm{loc}}\left(\omega, \omega^{\prime}\right) \delta\left(\boldsymbol{r}-\boldsymbol{r}^{\prime}\right)$,

where $\delta\left(\boldsymbol{r}-\boldsymbol{r}^{\prime}\right)$ is a three-dimensional Dirac delta. Therefore, we may proceed in a manner analogous to Eq. (2) to define:

$$
\begin{aligned}
\frac{\mathrm{d} f_{\text {loc }}}{\mathrm{d} \omega^{\prime} \mathrm{d} V^{\prime}} & =\int \mathrm{d} \omega \int_{\mathcal{V}} \mathrm{d} V \mathcal{N}(\omega, \boldsymbol{r}) p_{\text {loc }}\left(\omega, \omega^{\prime}\right) \delta\left(\boldsymbol{r}-\boldsymbol{r}^{\prime}\right) \\
& =\int \mathrm{d} \omega \mathcal{N}\left(\omega, \boldsymbol{r}^{\prime}\right) p_{\text {loc }}\left(\omega, \omega^{\prime}\right)
\end{aligned}
$$

To be able to calculate the effect of self-absorption on the local PL, including interference phenomena due to the particle size and shape, we need an explicit representation of the electromagnetic fields produced in each elementary emission process. We will represent PL emission as a distribution of oscillating electric dipoles: for each frequency interval $\mathrm{d} \omega^{\prime}$ we associate to each volume element $\mathrm{d} V^{\prime}$ in the dust particle a collection of incoherently oscillating electric dipoles, oriented in the direction $\mathrm{d} \Omega_{p}$. We will call this quantity, which has the dimensions of dipole moment per unit volume, unit frequency interval and unit solid angle

$$
\frac{\mathrm{d} \boldsymbol{p}}{\mathrm{d} \omega^{\prime} \mathrm{d} V^{\prime} \mathrm{d} \Omega_{p}}=\frac{\mathrm{d} \boldsymbol{p}_{0}}{\mathrm{~d} \omega^{\prime} \mathrm{d} V^{\prime} \mathrm{d} \Omega_{p}} \mathrm{e}^{-i \omega^{\prime} t} .
$$

This distribution of dipoles will be defined by requiring it to produce the local PL emission $\frac{\mathrm{d} f_{\mathrm{loc}}}{\mathrm{d} \omega^{\prime} \mathrm{d} V^{\prime}}$, as defined in Eq. (10).

Such a representation will be obviously appropriate if PL is indeed due to electric dipole permitted transitions, while a different representation, such as a density of (possibly higher order) electric or magnetic oscillating multipoles, would be more appropriate for a different transition. We remark, however, that in the case of absorption an adequate description can usually be obtained just in terms of the complex refractive index which, for non-magnetic materials, is determined only by the electric polarisability. There is no reason a priori why such a simplified representation should not be just as adequate to describe PL in the same material; however, in any case, using a more detailed representation would pose no significant conceptual problems, but merely complicate the practical calculations involved.

A single electric dipole $\boldsymbol{p}=\boldsymbol{p}_{0} \mathrm{e}^{-i \omega^{\prime} t}$, oscillating with frequency $\omega^{\prime}$ inside the grain, radiates a power given by:

$\mathcal{P}_{\text {dip }}=\frac{\omega^{\prime 4} \mu\left(\omega^{\prime}\right) n\left(\omega^{\prime}\right)\left\|p_{0}\right\|^{2}}{12 \pi c}$

where $n\left(\omega^{\prime}\right)$ is the real part of the refractive index $N_{1}$ and $\mu\left(\omega^{\prime}\right)$ is the magnetic permeability of the medium composing the particle. This is the expression given by Jackson (1998) for a dipole placed in vacuum, modified to be valid for arbitrary dielectric media, the difference being in the factor $n\left(\omega^{\prime}\right)$. We can use Eq. (12) to calculate the power and the photon emission rate per unit volume, unit frequency and unit solid angle stemming from our dipole distribution, to yield

$$
\frac{\mathrm{d} \mathcal{P}_{\operatorname{dip}}}{\mathrm{d} \omega^{\prime} \mathrm{d} V^{\prime} \mathrm{d} \Omega_{p}}=\frac{\omega^{\prime 4} \mu\left(\omega^{\prime}\right) n\left(\omega^{\prime}\right)}{12 \pi c}\left\|\frac{\mathrm{d} \boldsymbol{p}_{0}}{\mathrm{~d} \omega^{\prime} \mathrm{d} V^{\prime} \mathrm{d} \Omega_{p}}\right\|^{2}
$$

and

$$
\frac{\mathrm{d} f_{\text {dip }}}{\mathrm{d} \omega^{\prime} \mathrm{d} V^{\prime} \mathrm{d} \Omega_{p}}=\frac{\omega^{\prime 3} \mu\left(\omega^{\prime}\right) n\left(\omega^{\prime}\right)}{12 \pi \hbar c}\left\|\frac{\mathrm{d} \boldsymbol{p}_{0}}{\mathrm{~d} \omega^{\prime} \mathrm{d} V^{\prime} \mathrm{d} \Omega_{p}}\right\|^{2} .
$$

For this distribution of electric dipoles to represent the PL emission, we must require:

$$
\frac{\mathrm{d} f_{\text {dip }}}{\mathrm{d} \omega^{\prime} \mathrm{d} V^{\prime}}=\int \mathrm{d} \Omega_{p} \frac{\mathrm{d} f_{\text {dip }}}{\mathrm{d} \omega^{\prime} \mathrm{d} V^{\prime} \mathrm{d} \Omega_{p}}=\frac{\mathrm{d} f_{\text {loc }}}{\mathrm{d} \omega^{\prime} \mathrm{d} V^{\prime}} .
$$

The distribution of orientations of the dipoles can, in principle, be very complicated, containing detailed information on the microscopic structure of the material and a nontrivial correlation between the direction of the electric field of the exciting wave and the orientation of the excited emitting dipole. If e.g. the material is a molecular solid, this will include the distribution of the orientation of the molecules, the orientation of the transition dipole moment(s) relevant for the absorption, the orientation of the transition dipole moment(s) relevant for the PL emission. This can cause a very significant correlation between the direction of the electric field in the incoming wave and in the outgoing PL emission (Rusli et al. 1996). This effect is maximum if the exciting light is polarised to begin with, and if the transition dipole moment(s) relevant for the absorption and the transition dipole moment(s) relevant for the PL emission are parallel. This is true, in particular, if one considers PL emission excited by a polarised laser beam and examines PL emission at a frequency very close to that of excitation, maximising the probability that the fluorescent transition be the same that caused the absorption: in this case, the transition moments are obviously coincident. For astrophysical applications, however, we are interested in PL excited by natural light, hence non-monochromatic and unpolarised, in an amorphous molecular solid, such as the refractory organic residue which is thought to be produced by energetic processing of interstellar ices (see e.g. Greenberg \& Pirronello 1991). In such a material, there is obviously no preferential orientation of the molecules, which would otherwise show up as birefringence as well. As to the specific case of the Extended Red Emission, this is known to be excited in the UV and emitted in the visible (Smith \& Witt 2002). To wrap things up, in the organic clusters which make up the organic refractory residue, UV absorption is due to the superposition of a huge number of differently polarised electronic transitions, all of them contributing to the subsequent PL emission. For these reasons, we will here make the admittedly drastic simplification that any polarisation information be lost between absorption and the subsequent PL emission. The detailed study of possible polarisation effects will be dealt with in a subsequent paper.

In the particularly simple case in which the dipole distribution is isotropic, the integral over $\mathrm{d} \Omega_{p}$ amounts to a simple multiplication by $4 \pi$, hence from Eqs. (14) and (15) we obtain the relation:

$\left\|\frac{\mathrm{d} p_{0}}{\mathrm{~d} \omega^{\prime} \mathrm{d} V^{\prime} \mathrm{d} \Omega_{p}}\right\|^{2}=\frac{3 \hbar c}{\omega^{\prime 3} \mu\left(\omega^{\prime}\right) n\left(\omega^{\prime}\right)} \frac{\mathrm{d} f_{\text {loc }}}{\mathrm{d} \omega^{\prime} \mathrm{d} V^{\prime}}$.

Our approach closely resembles the semi-classical formalism of inelastic scattering by single molecules embedded in small 
particles, as developed in several papers (Chew et al. 1976a,b; Kerker et al. 1978a,b; Kerker \& Druger 1979; Wang et al. 1980; Videen et al. 1991; Hill et al. 1996; Pendleton \& Hill 1997). In this theory induced emission is represented in two separated steps. In the first, a molecule located at a particular position inside the grain is excited by the absorption of a photon at the incident frequency $\omega$. The second step is the emission of radiation at the shifted frequency $\omega^{\prime}$ by the active molecules at any location within the particle; this emission is described by the electromagnetic field of an induced dipole placed at the same location.

We now need to determine the electromagnetic fields leaving the dust grain, given an oscillating dipole moment located at a specified position inside it. This problem is completely analogous to that of calculating absorption and scattering by a dust particle, given its optical properties and an incoming plane wave. The transmitted fields outside the particle are expressed as $\left(\boldsymbol{E}_{2}, \boldsymbol{H}_{2}\right)$, while the internal fields $\left(\boldsymbol{E}_{1}, \boldsymbol{H}_{1}\right)$ are decomposed into the sum:

$\boldsymbol{E}_{1}=\boldsymbol{E}_{\mathrm{d}}+\boldsymbol{E}_{\mathrm{s}}, \quad \boldsymbol{H}_{1}=\boldsymbol{H}_{\mathrm{d}}+\boldsymbol{H}_{\mathrm{s}}$,

between the fields $\left(\boldsymbol{E}_{\mathrm{d}}, \boldsymbol{H}_{\mathrm{d}}\right)$ produced by the oscillating dipole and the scattered fields $\left(\boldsymbol{E}_{\mathrm{s}}, \boldsymbol{H}_{\mathrm{s}}\right)$ inside the particle. $\boldsymbol{E}_{\mathrm{d}}$ and $\boldsymbol{H}_{\mathrm{d}}$ are the fields produced by an oscillating dipole embedded in an unlimited medium characterised by the refractive index $N_{1}$, and therefore include the contribution by the induced dipoles in the medium. The fields $\boldsymbol{E}_{\mathrm{s}}$ and $\boldsymbol{H}_{\mathrm{s}}$ are essentially due to reflections at the boundary of the particle. Indeed, as shown in Appendix A, when the material of the sphere and that of the surrounding medium have the same refractive index these scattered fields vanish identically.

We are ultimately interested in the total power irradiated by the particle into a unit solid angle about a given direction. To obtain it, we first evaluate the instantaneous Poynting vector $S$ from the outgoing fields $\left(\boldsymbol{E}_{2}, \boldsymbol{H}_{2}\right)$, under the far-field approximation; then a time average and a sum over all possible orientations of the dipole are performed. This is the power $\frac{\mathrm{d} \mathcal{P}_{\mathrm{pl}}}{\mathrm{d} \Omega \mathrm{d} \omega^{\prime} \mathrm{d} V^{\prime}}$ emitted per unit solid angle around a given direction, after the radiation emitted by the volume element $\mathrm{d} V^{\prime}$ escapes the particle. As derived in detail in Appendix A, if the dipole distribution is isotropic the above quantity turns out to be given by

$\frac{\mathrm{d} \mathcal{P}_{\mathrm{pl}}}{\mathrm{d} \Omega \mathrm{d} \omega^{\prime} \mathrm{d} V^{\prime}}=4 \pi \frac{\mathrm{d} \mathcal{P}_{\mathrm{dip}}}{\mathrm{d} \omega^{\prime} \mathrm{d} V^{\prime} \mathrm{d} \Omega_{p}} \frac{\mathrm{d} \mathcal{F}}{\mathrm{d} \Omega}$.

The factor $\frac{\mathrm{d} \mathcal{F}}{\mathrm{d} \Omega}$ contains, in a sense, all the effects of the dust grain on the single PL photon emitted inside it, as indeed it modulates the isotropic power emitted inside the particle, affecting the angular and spectral distribution of the emerging PL. Equations (10) through (16) can be combined with Eq. (18) above to yield:

$$
\frac{\mathrm{d} \mathcal{P}_{\mathrm{pl}}}{\mathrm{d} \Omega \mathrm{d} \omega^{\prime} \mathrm{d} V}=\hbar \omega^{\prime} \frac{\mathrm{d} f_{\text {loc }}}{\mathrm{d} \omega^{\prime} \mathrm{d} V^{\prime}} \frac{\mathrm{d} \mathcal{F}}{\mathrm{d} \Omega} .
$$

To simplify the subsequent formalism, we henceforth consider monochromatic excitation at angular frequency $\omega$. This implies no loss of generality, since the general case can still be obtained by superposition. Eq. (19) is thus simplified to:

$\frac{\mathrm{d} \mathcal{P}_{\mathrm{pl}}}{\mathrm{d} \Omega \mathrm{d} \omega^{\prime} \mathrm{d} V^{\prime}}=\hbar \omega^{\prime} p_{\text {loc }}\left(\omega, \omega^{\prime}\right) \mathcal{N}\left(\omega, \boldsymbol{r}^{\prime}\right) \frac{\mathrm{d} \mathcal{F}}{\mathrm{d} \Omega}$.

Integrating the previous equation in $\mathrm{d} V^{\prime}$ over the volume of the particle $\mathcal{V}$ we obtain the total PL power per unit frequency and unit solid angle in a given direction:

$\frac{\mathrm{d} \mathcal{P}_{\mathrm{pl}}}{\mathrm{d} \Omega \mathrm{d} \omega^{\prime}}=\hbar \omega^{\prime} p_{\text {loc }}\left(\omega, \omega^{\prime}\right) \int_{\mathcal{V}} \mathcal{N}\left(\omega, \boldsymbol{r}^{\prime}\right) \frac{\mathrm{d} \mathcal{F}}{\mathrm{d} \Omega}$,

following absorption at frequency $\omega$.

In light scattering theory the extinction properties of small particles are usually expressed in terms of the extinction cross-section $C_{\text {ext }}$, which is the sum of the absorption crosssection $C_{\text {abs }}$ and the scattering cross-section $C_{\text {sca }}$ (Bohren \& Huffman 1998). To follow this convention and to clearly separate the dependence of $\mathcal{N}\left(\omega, \boldsymbol{r}^{\prime}\right)$ on the position inside the particle, we express the source function $\mathcal{E}(\boldsymbol{r}, \omega)$ in Eq. (1) as the irradiance $^{2} I(\omega)$, times $\frac{\mathrm{d} C_{\text {abs }}}{\mathrm{d} V}$ :

$\mathcal{N}\left(\omega, \boldsymbol{r}^{\prime}\right)=\frac{\mathcal{E}(\boldsymbol{r}, \omega)}{\hbar \omega}=\frac{I(\omega)}{\hbar \omega} \frac{\mathrm{d} C_{\mathrm{abs}}}{\mathrm{d} V}$

where $\frac{\mathrm{d} C_{\mathrm{abs}}}{\mathrm{d} V}$ can be seen as the contribution by the volume element $\mathrm{d} V$ to the total absorption cross-section $C_{\mathrm{abs}}$ of the particle. Therefore, Eq. (21) reduces to:

$\frac{\mathrm{d} \mathcal{P}_{\mathrm{pl}}}{\mathrm{d} \Omega \mathrm{d} \omega^{\prime}}=\frac{\omega^{\prime}}{\omega} I(\omega) p_{\text {loc }}\left(\omega, \omega^{\prime}\right) \int_{\mathcal{V}} \frac{\mathrm{d} C_{\mathrm{abs}}}{\mathrm{d} V} \frac{\mathrm{d} \mathcal{F}}{\mathrm{d} \Omega} \mathrm{d} V$.

The ratio of $\frac{\mathrm{d} \mathcal{P}_{\mathrm{pl}}}{\mathrm{d} \Omega \mathrm{d} \omega^{\prime}}$ to $I(\omega)$ is a quantity with dimensions of area per unit solid angle, per unit frequency which we define as the differential cross-section for PL:

$\frac{\mathrm{d} C_{\mathrm{pl}}}{\mathrm{d} \Omega \mathrm{d} \omega^{\prime}}=\frac{\mathrm{d} \mathcal{P}_{\mathrm{pl}}}{\mathrm{d} \Omega \mathrm{d} \omega^{\prime}} \frac{1}{I(\omega)}$.

Physically it expresses the angular distribution of the light emitted by the particle: the amount of light (per unit incident irradiance) emitted at frequency $\omega^{\prime}$ by the particle, into a unit solid angle about a given direction. This definition is similar to the one of the differential scattering cross section $\frac{\mathrm{d} C_{\text {sca }}}{\mathrm{d} \Omega}$, commonly used in light scattering theory (see e.g., Bohren \& Huffman 1998). We thus write:

$\frac{\mathrm{d} C_{\mathrm{pl}}}{\mathrm{d} \Omega \mathrm{d} \omega^{\prime}}=\frac{\omega^{\prime}}{\omega} p_{\text {loc }}\left(\omega, \omega^{\prime}\right) \int_{\mathcal{V}} \frac{\mathrm{d} C_{\mathrm{abs}}}{\mathrm{d} V} \frac{\mathrm{d} \mathcal{F}}{\mathrm{d} \Omega} \mathrm{d} V$.

The treatment we presented so far is very general, and can be applied, in principle, to any case for which the electric and magnetic fields induced inside the particle by an incident light beam can be calculated. In particular we can

1. apply the model to a known experimental configuration, in which $I(\omega), \frac{\mathrm{d} \mathcal{P}_{\mathrm{pl}}}{\mathrm{d} \Omega \mathrm{d} \omega^{\prime}}, \frac{\mathrm{d} \mathcal{F}}{\mathrm{d} \Omega}$ and $\frac{\mathrm{d} C_{\mathrm{abs}}}{\mathrm{d} V}$ can all be measured or
computed, in order to derive $p_{\text {loc }}\left(\omega, \omega^{\prime}\right)$;

${ }^{2} I(\omega)$ is the magnitude of the Poynting vector of the incident field; its dimensions are energy per unit area and unit time. 
2. apply the model to the (astrophysically relevant) small dust particle we are interested in, for which we know $p_{\text {loc }}\left(\omega, \omega^{\prime}\right)$ from the first step, we can compute $\frac{\mathrm{d} \mathcal{F}}{\mathrm{d} \Omega}$ and $\frac{\mathrm{d} C_{\mathrm{abs}}}{\mathrm{d} V}$ and, assuming that we know $I(\omega)$, obtain $\frac{\mathrm{d} \mathcal{P}_{\mathrm{pl}}}{\mathrm{d} \Omega \mathrm{d} \omega^{\prime}}$.

In equations, the first step can be written as

$$
p_{\mathrm{loc}}\left(\omega, \omega^{\prime}\right)=\frac{\omega}{\omega^{\prime}} \cdot \frac{\left(\frac{\mathrm{d} C_{\mathrm{pl}}}{\mathrm{d} \Omega \mathrm{d} \omega^{\prime}}\right)_{\mathrm{lab}}}{\left(\int_{\mathcal{V}} \frac{\mathrm{d} C_{\mathrm{abs}}}{\mathrm{d} V} \frac{\mathrm{F}}{\mathrm{d} \Omega} \mathrm{d} V\right)_{\mathrm{lab}}},
$$

while the second step can be written as

$$
\left(\frac{\mathrm{d} C_{\mathrm{pl}}}{\mathrm{d} \Omega \mathrm{d} \omega^{\prime}}\right)_{\text {dust }}=\left(\frac{\mathrm{d} C_{\mathrm{pl}}}{\mathrm{d} \Omega \mathrm{d} \omega^{\prime}}\right)_{\mathrm{lab}} \frac{\left(\int_{\mathcal{V}} \frac{\mathrm{d} C_{\mathrm{abs}}}{\mathrm{d} V} \frac{\mathrm{d} \mathcal{F}}{\mathrm{d} \Omega} \mathrm{d} V\right)_{\text {dust }} .}{\left.\int_{\mathcal{V}} \frac{\mathrm{d} C_{\mathrm{abs}}}{\mathrm{d} V} \frac{\mathrm{d} \mathcal{F}}{\mathrm{d} \Omega} \mathrm{d} V\right)_{\text {lab }}} .
$$

All of the small particle effects, including the specific size of the particle, their angular dependence and their dependence on the complex refractive index of the material are completely contained in the factor multiplying $\left(\frac{\mathrm{d} C_{\mathrm{pl}}}{\mathrm{d} \Omega \mathrm{d} \omega^{\prime}}\right)_{\mathrm{lab}}$ on the right hand side of the above equation. Hence these two steps, taken together, provide a general recipe to extrapolate laboratory PL results, obtained from bulk samples, to small dust particles, under the assumption that PL excitation is local and the resulting emission isotropic, which was the aim of the present work.

The above Eq. (27), derived for monochromatic incident light and a specific dust particle shape and size, can be straightforwardly generalised for a distribution $n(a)$ of particles and non-monochromatic incident light. In this case one simply gets

$$
\frac{\mathrm{d} \mathcal{P}_{\mathrm{pl}}}{\mathrm{d} \Omega \mathrm{d} \omega^{\prime}}=\int \mathrm{d} a \int \mathrm{d} \omega n(a) I(\omega)\left(\frac{\mathrm{d} C_{\mathrm{pl}}}{\mathrm{d} \Omega \mathrm{d} \omega^{\prime}}\right)_{\omega} .
$$

\subsection{Local PL from a homogeneous sphere}

To provide a simple, practical "proof of concept" implementation of our model, while still able to yield some useful physical insight in the study of PL from interstellar dust grains, we consider a spherical, homogeneous particle illuminated by an unpolarised, parallel light beam. This enables us to make use of the standard Lorenz-Mie theory to describe the absorption and to derive analytical results for the resulting PL. This simple case can also be easily adapted to model a realistic laboratory configuration, hence providing the foundation for both the first and the second steps outlined in the previous section.

To exploit the symmetry of the problem we expand all of the fields as series of vector spherical harmonics (VSHs), which can be shown to be orthogonal and complete for transverse waves (see e.g., Bohren \& Huffman 1998). As usual, the expansion coefficients can be derived by imposing the continuity of tangential components of the fields at the boundary surface between the particle and the surrounding medium, and using the orthogonality properties of the VSHs. All of the relevant formulae obtained for this case are presented in Appendix A.
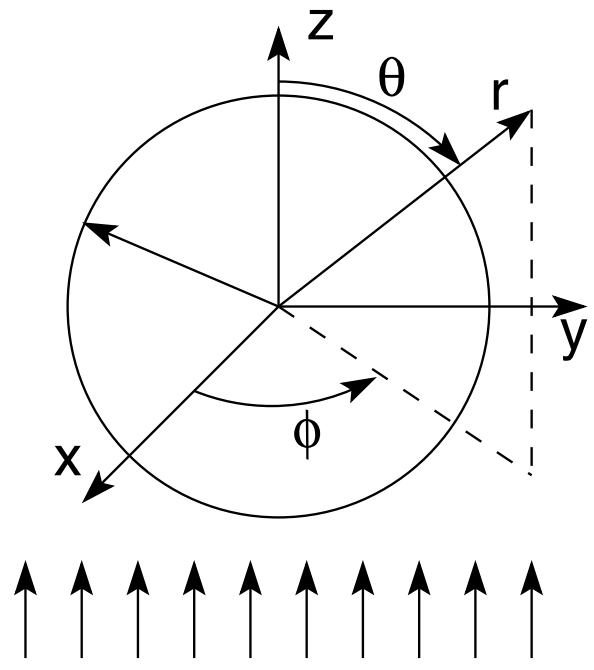

Fig. 1. Representation of the chosen coordinate system.

We refer the reader interested in the details of the full analytical derivation to Malloci (2003). We expressed the angular dependence of both $\frac{\mathrm{d} C_{\mathrm{abs}}}{\mathrm{d} V}$ and $\frac{\mathrm{d} \mathcal{F}}{\mathrm{d} \Omega}$ into Eq. (33) with the help of the generalised spherical functions (GSFs) $P_{m, n}^{l}$ (Hovenier $\&$ Van der Mee 1983). As expected from the symmetry of the problem, we obtain

$\frac{\mathrm{d} C_{\mathrm{pl}}}{\mathrm{d} \Omega \mathrm{d} \omega^{\prime}}=\frac{\omega^{\prime}}{\omega} p_{\mathrm{loc}}\left(\omega, \omega^{\prime}\right) \sum_{t} \gamma_{t}\left(a, \omega, \omega^{\prime}\right) P_{t}(\cos \theta)$,

where the angular dependence on the angle $\theta$ between the direction of incoming light and the direction of the PL light (see Fig. 1) is expressed in a series of Legendre polynomials $P_{t}(\cos \theta)$. The explicit expression for the $\gamma_{t}$ coefficients can be derived with some algebraic labour as shown in Appendix A. They depend explicitly on the particle size and on the refractive index $N_{1}$ and, through the latter, implicitly on $\omega$ and $\omega^{\prime}$.

In turn, according to the first step outlined in the previous section, we can express $p_{\text {loc }}\left(\omega, \omega^{\prime}\right)$ in terms of the experimentally measured PL yield $\frac{\mathrm{d} \eta_{\text {lab }}}{\mathrm{d} \Omega \mathrm{d} \omega^{\prime}}$ :

$\frac{\omega^{\prime}}{\omega} p_{\text {loc }}\left(\omega, \omega^{\prime}\right)=\frac{\mathrm{d} \eta_{\text {lab }}}{\mathrm{d} \Omega \mathrm{d} \omega^{\prime}} \cdot \frac{1}{g\left(\omega, \omega^{\prime}, \theta_{\mathrm{d}}\right)}$,

where

$\frac{\mathrm{d} \eta_{\text {lab }}}{\mathrm{d} \Omega \mathrm{d} \omega^{\prime}}=\frac{\left(\frac{\mathrm{d} \mathcal{P}_{\mathrm{pl}}}{\mathrm{d} \Omega \mathrm{d} \omega^{\prime}}\right)_{\mathrm{lab}}}{\mathcal{P}_{\mathrm{abs}}}$

is the total PL yield, i.e. the ratio between the measured PL power per unit solid angle and unit frequency in a given direction, as shown in the experimental configuration depicted in Fig. 2, and the power absorbed by the sample from the monochromatic incident exciting light beam. The term $g\left(\omega, \omega^{\prime}, \theta_{\mathrm{d}}\right)$ includes all the gory details of light propagation and self-absorption in the macroscopic laboratory sample, which are obviously dependent on $\omega, \omega^{\prime}$ and the experimental configuration, explicitly represented in this case by the angle $\theta_{\mathrm{d}}$, as defined in Fig. 2. Of course, the function $g$ will be 


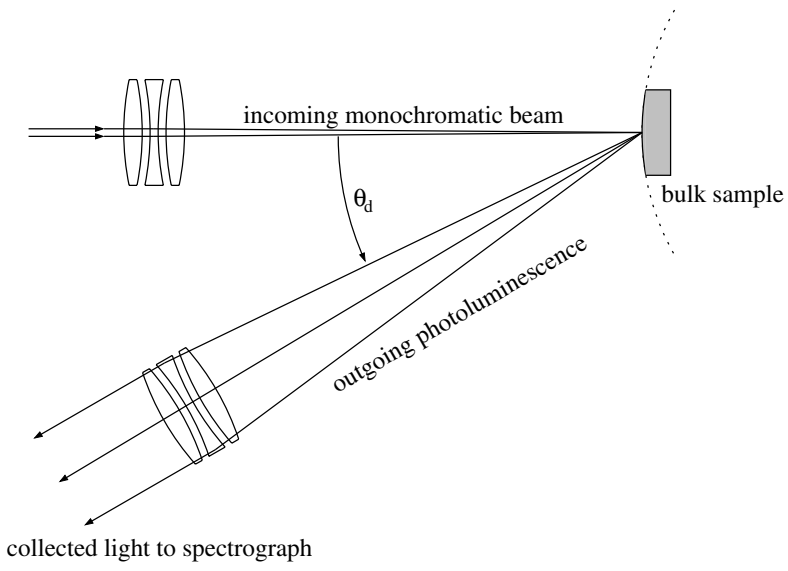

Fig. 2. Schematic description of the experimental setup to measure the PL yield $\frac{\mathrm{d} \eta_{\text {lab }}}{\mathrm{d} \Omega \mathrm{d} \omega^{\prime}}$ from a bulk sample and hence obtain $p_{\text {loc }}\left(\omega, \omega^{\prime}\right)$ in the local approximation. The sample can be seen as a portion of a sphere of very large radius, represented by the dotted outline.

different for different specific experimental configurations, possibly depend on different parameters, and will thus need to be calculated on a case by case basis. The derivation of $g\left(\omega, \omega^{\prime}, \theta_{\mathrm{d}}\right)$ for the specific case implemented here can be found in the third part of Appendix A; for more details we again refer the reader to Malloci (2003). Figure 3 shows its behaviour for two different values of $\theta_{\mathrm{d}}$ and three different excitation frequencies.

We can now combine Eqs. (29) and (30) to obtain

$\frac{\mathrm{d} C_{\mathrm{pl}}}{\mathrm{d} \Omega \mathrm{d} \omega^{\prime}}=\frac{\mathrm{d} \eta_{\mathrm{lab}}}{\mathrm{d} \Omega \mathrm{d} \omega^{\prime}} \cdot \mathcal{A}\left(\omega, \omega^{\prime}, a, \theta, \theta_{\mathrm{d}}\right)$,

where the form factor $\mathcal{A}\left(\omega, \omega^{\prime}, a, \theta, \theta_{\mathrm{d}}\right)$ (with dimensions of an area) is given by:

$\mathcal{A}\left(\omega, \omega^{\prime}, a, \theta, \theta_{\mathrm{d}}\right)=\frac{1}{g\left(\omega, \omega^{\prime}, \theta_{\mathrm{d}}\right)} \sum_{t} \gamma_{t}\left(a, \omega, \omega^{\prime}\right) P_{t}(\cos \theta)$.

Equations (32) and (33) show two properties:

- $\frac{\mathrm{d} C_{\mathrm{pl}}}{\mathrm{d} \Omega \mathrm{d} \omega^{\prime}}$ is the product of the experimentally measured PL yield times the "form factor" $\mathcal{A}\left(\omega, \omega^{\prime}, a, \theta, \theta_{\mathrm{d}}\right)$ which wholly contains all the small particle effects, including their angular dependence and their dependence on the complex refractive index of the material;

- the angular dependence of $\frac{\mathrm{d} C_{\mathrm{pl}}}{\mathrm{d} \Omega \mathrm{d} \omega^{\prime}}$ is expressed analytically in terms of a simple expansion in Legendre polynomials $P_{t}(\cos \theta)$.

Equation (32) provides the promised link between the experimental measurement of PL, as performed on a macroscopic sample, and the expected PL spectrum for the same material "ground" into small spheres. The computation of the expansion coefficients $\gamma_{t}$ involves a single numerical integration in the radial dimension of the dust particle. For any practical purposes, this series can be truncated at a finite, relatively small number of terms (Bohren \& Huffman 1998).

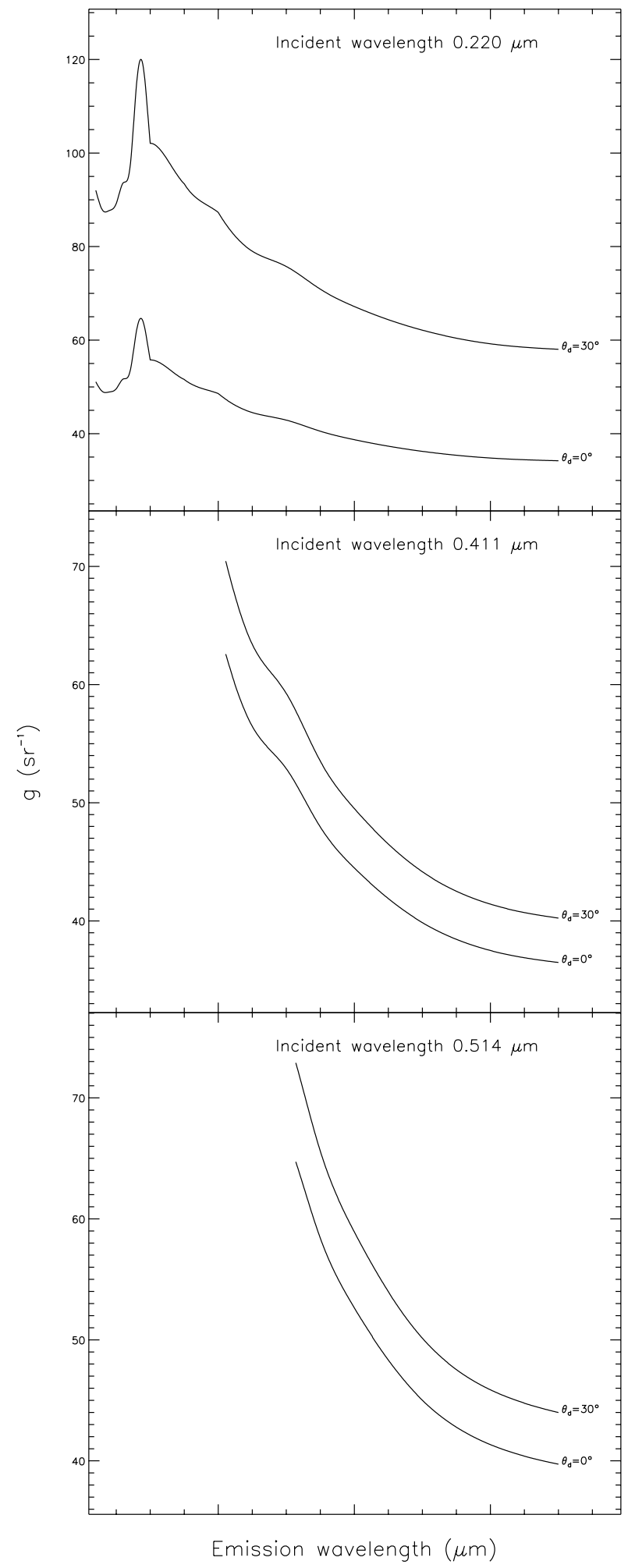

Fig. 3. The function $g\left(\omega, \omega^{\prime}\right)$ computed in the local approximation for the experimental setup depicted in Fig. 2, for three fixed incident wavelengths and two different values of the detector angle $\theta_{\mathrm{d}}$.

\subsection{Secondary $P L$}

In the practical implementation we presented here, we made the simplifying approximation to only consider primary PL, i.e. the emission of photons following the absorption of one 

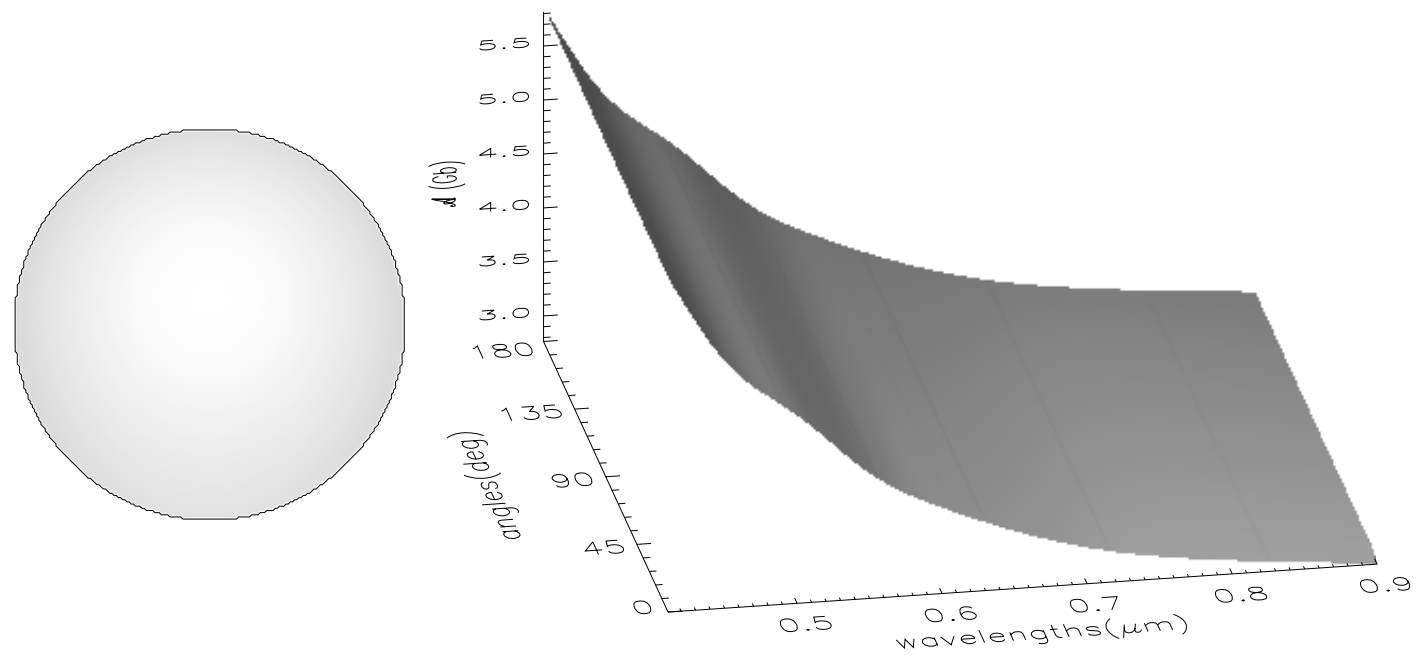

Fig. 4. Sample or1: incident wavelength $0.411 \mu \mathrm{m}$, sphere radius $0.05 \mu \mathrm{m}(x \sim 0.8$, close to the Rayleigh limit).

photon from the incoming external field. We solved the well known problem of the absorption of light from an incident unpolarised wave and expressed the source function as proportional to the divergence of the resulting Poynting vector inside the particle. However, $\mathcal{N}(\boldsymbol{r}, \omega)$ should also include a contribution from self-absorbed photons, as computed from the divergence of the Poynting vector stemming from the PL itself. This contribution to $\mathcal{N}(\boldsymbol{r}, \omega)$ gives rise to secondary PL, i.e. the emission of a photon following the self-absorption of a previous PL photon. This does not formally affect the derivation above at all, except for one point: one should consider the contribution of self-absorbed photons to the source function, which is obtained from the divergence of the Poynting vector associated to the internal field $\left(\boldsymbol{E}_{1}, \boldsymbol{H}_{1}\right)$, of Eq. (17). As a consequence of this inclusion, PL at any given wavelength will be related to the PL and optical properties of the material at all smaller wavelengths, making practical calculations much more difficult. A perturbative approach is always possible, in which one considers, as a first approximation, just the contribution to the source function from the incident exciting field, computes the resulting PL and the resulting contribution of selfabsorption to the source function, and repeats the calculations in a self-consistent way until the desired accuracy is reached. However, each subsequent correction will be of the order of the PL yield times the previous one; therefore, for any reasonable PL yield, the zero order approximation to completely neglect secondary PL will already be a good one, and any corrections beyond the first order will be extremely small.

From a qualitative point of view, the effect of secondary PL is obviously in the direction of increasing the yield, this increase being necessarily in the form of lower energy photons, moving the peak of the spectrum slightly to the red.

\section{Numerical results}

We present here the numerical results of the application of our local PL model to the specific case of a spherical, homogeneous dust particle, considering only primary PL. We used the optical properties of processed organic refractory residues, in a form as expected in the diffuse interstellar medium, as given by Jenniskens (1993). In particular, we used the optical constants corresponding to two different organic refractory residues subjected to energetic processing, here labelled or1 and or2, the latter being the more heavily processed one. The outer medium was assumed to be the vacuum.

Figures 4 through 11 display a sample of the results, relative to excitation with different monochromatic exciting wavelengths, namely $220 \mathrm{~nm}, 411 \mathrm{~nm}$ and $514 \mathrm{~nm}$; the sphere radius is taken to be in the range $50-2050 \mathrm{~nm}$ and two sets of complex refractive indices were considered, corresponding to organic refractory residues obtained by UV irradiation of laboratory analogues of interstellar ices at different doses of absorbed energy. While we sampled a much larger grid of the parameter space, these results suffice to clearly demonstrate the main effects of self-absorption on the expected PL, and the impact on them of each parameter. They are illustrated with reference to the geometric configuration depicted in Fig. 1. The direction of propagation of the incident light defines the $z$ axis, the forward direction. The angle expressing the position of the detector in the laboratory setup assumed (see Fig. 2) is taken to be $\theta_{\mathrm{d}}=30^{\circ}$. Each figure shows at the left the distribution of the absorbed energy inside the sphere, expressed in terms of the absorption cross section per unit volume, evaluated through Eq. (A.1), normalised to the total $C_{\mathrm{abs}}$, computed using Eq. (A.2). The contour plots show the distribution of the locally absorbed energy in a plane containing the $z$-axis (cf. Fig. 1). Given the symmetry of the problem, if the exciting light is unpolarised the absorption pattern is the same in any such plane.

The box at the right represents the form factor $\mathcal{A}\left(\omega, \omega^{\prime}, a, \theta, \theta_{\mathrm{d}}\right)$, as a function of the angle $\theta$ between the incident beam and the direction of observation and of the emission wavelength $\lambda^{\prime}=2 \pi c / \omega^{\prime}$. Therefore, these three-dimensional plots offer an explicit visual representation of the impact of particle size effects in modulating the spectral distribution of the PL observed in laboratory experiments. The form factor $\mathcal{A}\left(\omega, \omega^{\prime}, a, \theta, \theta_{\mathrm{d}}\right)$ is expressed in gigabarns $\left(1 \mathrm{~Gb}=10^{-15} \mathrm{~cm}^{2}\right)$. 

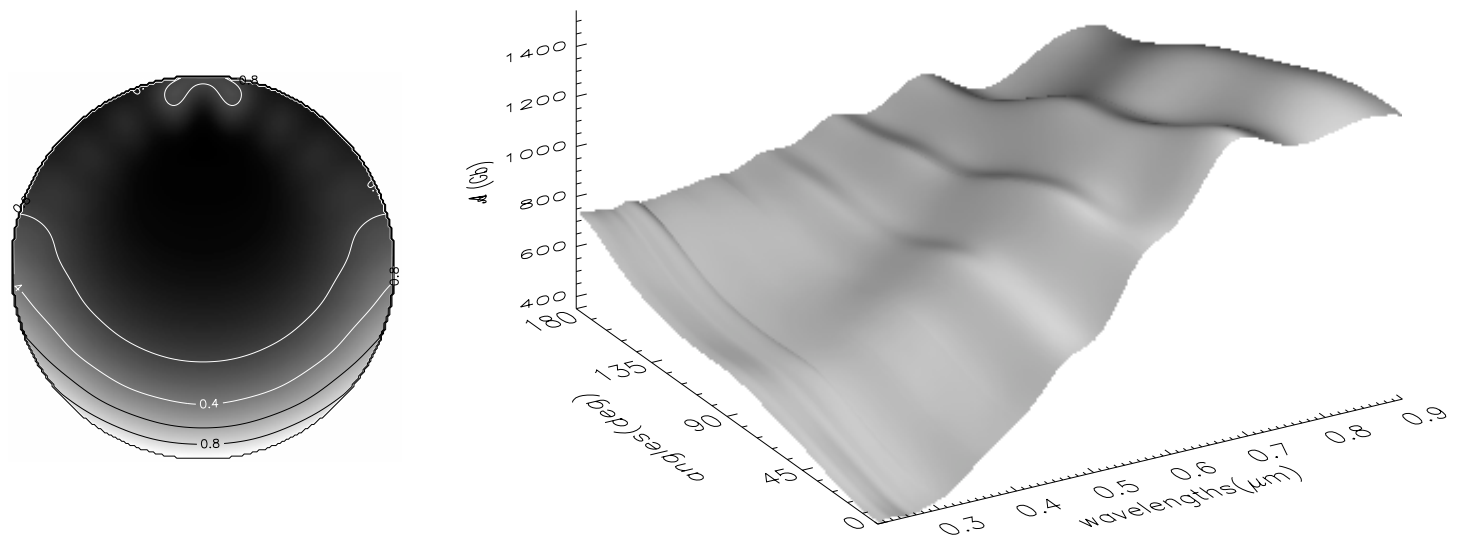

Fig. 5. Sample or1: incident wavelength $0.220 \mu \mathrm{m}$, sphere radius $0.35 \mu \mathrm{m}(x \sim 10.0)$.


Fig. 6. Sample or 1: incident wavelength $0.411 \mu \mathrm{m}$, sphere radius $0.35 \mu \mathrm{m}(x \sim 5.4)$.
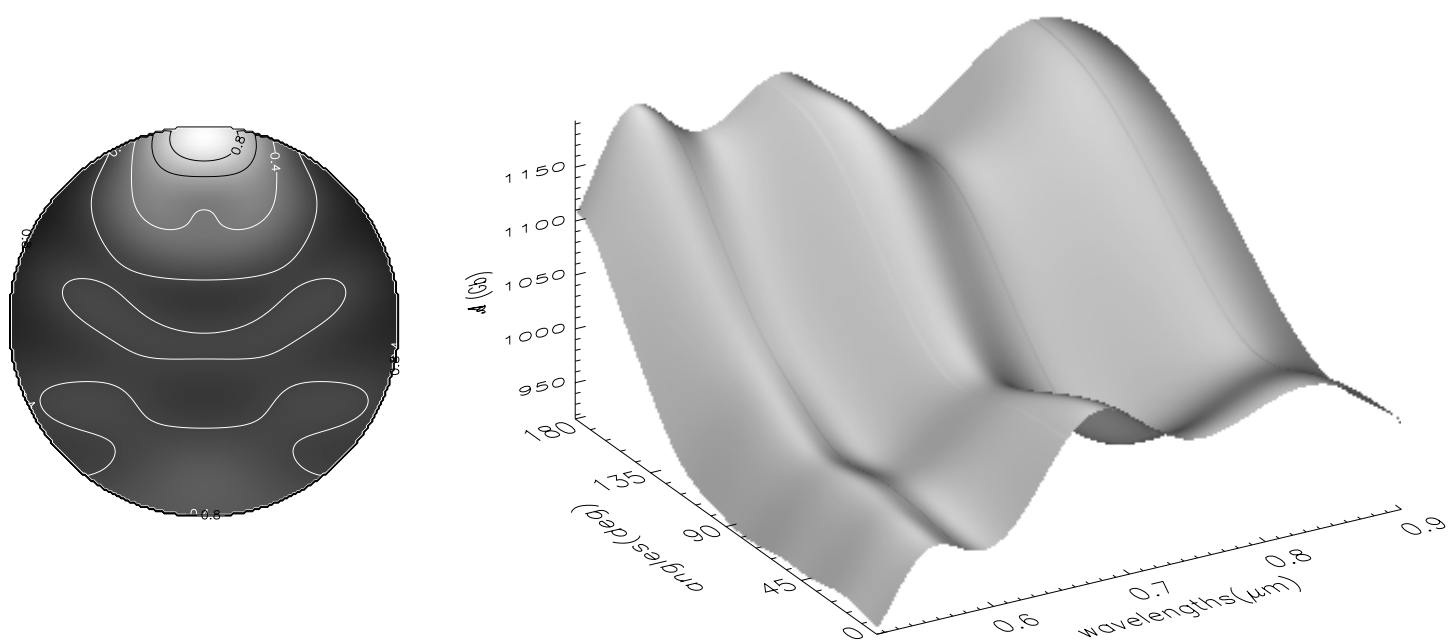

Fig. 7. Sample or1: incident wavelength $0.514 \mu \mathrm{m}$, sphere radius $0.35 \mu \mathrm{m}(x \sim 4.3)$.

\section{Discussion}

The distribution of the locally absorbed energy inside a particle is well known to be a complicated function of the position (Kerker 1973; Dusel et al. 1976). Our contour plots of the absorbed energy distribution correspond to increasing particle radii. As expected, featureless absorption is observed for very small size parameters (defined as $\omega a / c$ ), while for increasing $a$, the absorption shows an increasingly structured pattern.
For the optical properties of the sample or 1 considered in this work there are cases in which the absorption is larger on the side opposite to the illuminated one. At large size parameters absorption is increasingly concentrated on the side where the particle is irradiated, as expected when approaching the geometrical optics limit.

This richly structured absorption pattern governs the behaviour of $\mathcal{A}\left(\omega, \omega^{\prime}, a, \theta, \theta_{\mathrm{d}}\right)$, which turns out to strongly depend on the emitted wavelength $\lambda^{\prime}$ and on the angle $\theta$. This 

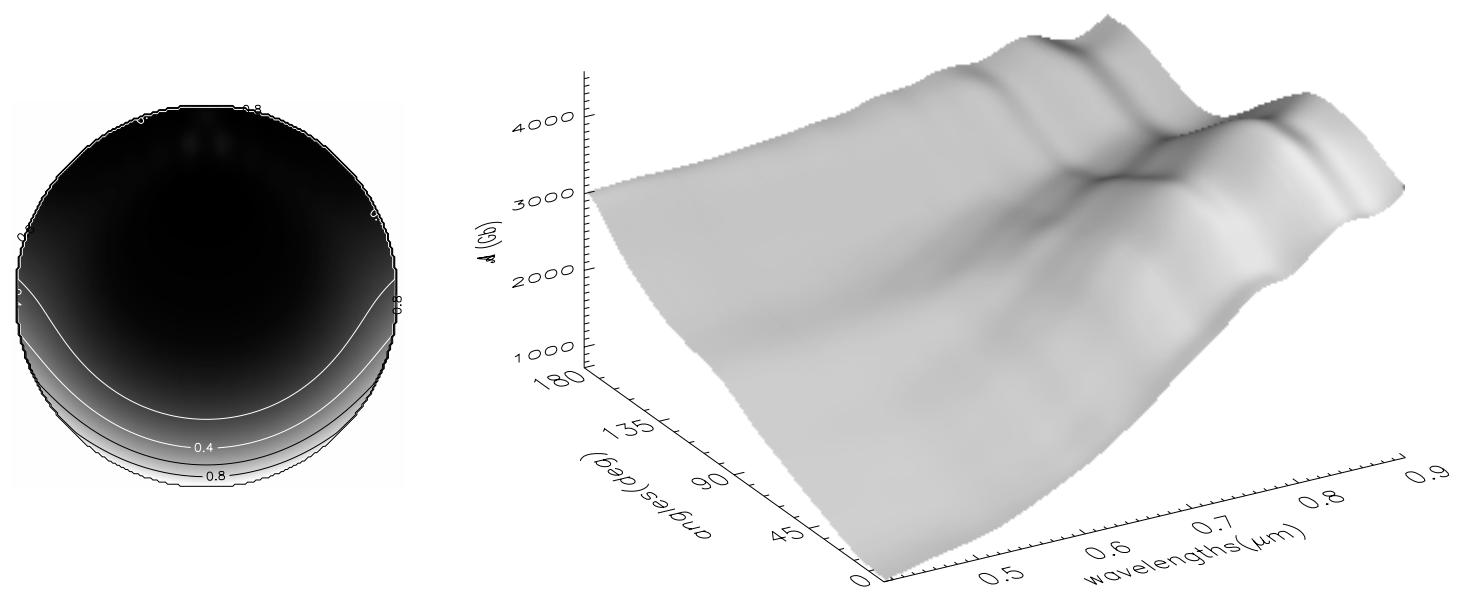

Fig. 8. Sample or1: incident wavelength $0.411 \mu \mathrm{m}$, sphere radius $0.95 \mu \mathrm{m}(x \sim 14.5)$.
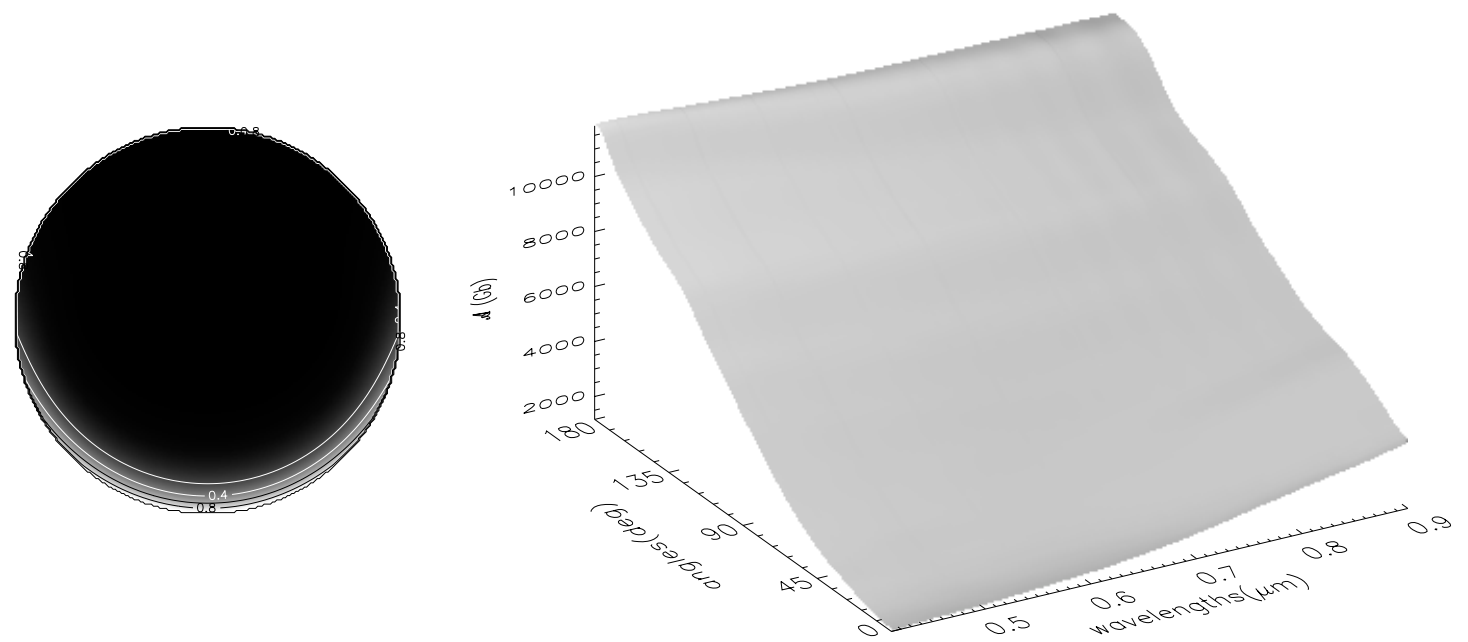

Fig. 9. Sample or1: incident wavelength $0.411 \mu \mathrm{m}$, sphere radius $2.05 \mu \mathrm{m}(x \sim 31.1$, approaching geometric optics limit).

vindicates our initial Ansatz that geometry effects are able to considerably modify the spectral distribution of the PL observed in laboratory experiments. In particular, the $\lambda^{\prime}$ dependence is conspicuous for any particle sizes: in the Rayleigh limit $(a=0.05 \mu \mathrm{m}$ and size parameters of about 0.8 and 0.6 for $\lambda=411 \mathrm{~nm}$ and $514 \mathrm{~nm}$, respectively) $\mathcal{A}\left(\omega, \omega^{\prime}, a, \theta, \theta_{\mathrm{d}}\right)$ changes by a factor of about 2-3 for $\lambda^{\prime}$ between $0.4 \mu \mathrm{m}$ and $0.9 \mu \mathrm{m}$, becoming even more marked for larger particles all the way up to the geometrical optics limit at $2.05 \mu \mathrm{m}$ (with size parameters of about 31.3 for $\lambda=411 \mathrm{~nm}$ and 25.1 for $\lambda=514 \mathrm{~nm}$ ).

As to the angular dependence, the results show an increasing variation for growing size parameters; the astrophysical implications are discussed in a subsequent paper (Mulas et al. 2004).

When the incident wavelength is comparable to the particle dimensions, $\mathcal{A}\left(\omega, \omega^{\prime}, a, \theta, \theta_{\mathrm{d}}\right)$ displays an oscillatory behaviour, which tends to be damped in the geometrical optics limit. This is not unexpected, as it is very similar to what one observes for extinction (see for example Fig. 4.6 on page 105 of Bohren \& Huffman (1998), which shows an interference structure showing a series of alternating broad maxima and minima, with weaker and weaker oscillations around the expected asymptotic value for increasingly large size parameters).

It should be noted that the positions of peaks and valleys, for a chosen material, depend on particle size and on exciting wavelength; therefore, the small scale interference structure is likely to be washed out if one observes a broad distribution of particle radii and/or if their PL is excited by non-monochromatic light with a broad spectrum. In the most generic case of non-monochromatic light and a distribution $n(a)$ of particle sizes we may rewrite Eq. (28) as

$$
\begin{aligned}
\frac{\mathrm{d} \mathcal{P}_{\mathrm{pl}}}{\mathrm{d} \Omega \mathrm{d} \omega^{\prime}} & =\int \mathrm{d} a \int \mathrm{d} \omega n(a) I(\omega)\left(\frac{\mathrm{d} C_{\mathrm{pl}}}{\mathrm{d} \Omega \mathrm{d} \omega^{\prime}}\right)_{\omega} \\
& =\int \mathrm{d} a n(a) \int \mathrm{d} \omega I(\omega) \mathcal{A}\left(\omega, \omega^{\prime}, a, \theta, \theta_{\mathrm{d}}\right)\left(\frac{\mathrm{d} \eta_{\mathrm{lab}}}{\mathrm{d} \Omega \mathrm{d} \omega^{\prime}}\right)_{\omega} \\
& =\sum_{t} P_{t}(\cos \theta) \int \mathrm{d} \omega \frac{I(\omega)}{g\left(\omega, \omega^{\prime}\right)}\left(\frac{\mathrm{d} \eta_{\mathrm{lab}}}{\mathrm{d} \Omega \mathrm{d} \omega^{\prime}}\right)_{\omega} \int \mathrm{d} a n(a) \gamma_{t}
\end{aligned}
$$

which retains the explicitly analytical angular dependence of the emitted power $\frac{\mathrm{d} \mathcal{P}_{\mathrm{pl}}}{\mathrm{d} \Omega \mathrm{d} \omega^{\prime}}$, expanded in a (conveniently truncated) series in $P_{t}(\cos \theta)$ (cf. Appendix A for more details). 

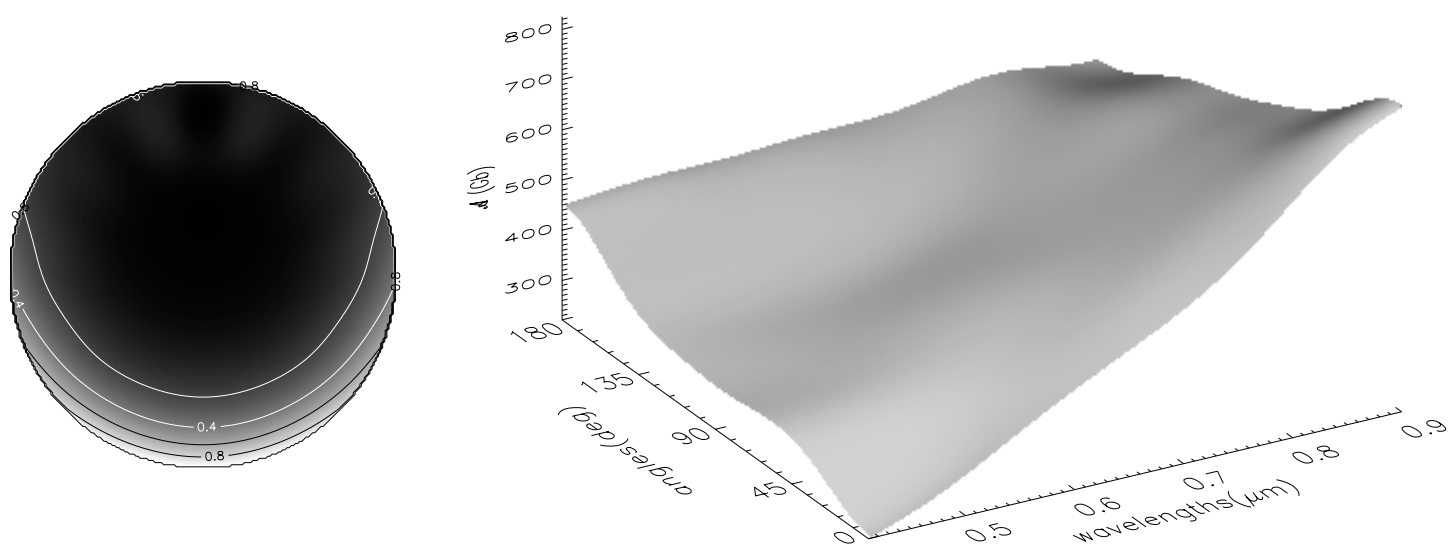

Fig. 10. Sample or2: incident wavelength $0.411 \mu \mathrm{m}$, sphere radius $0.35 \mu \mathrm{m}(x \sim 4.3)$.
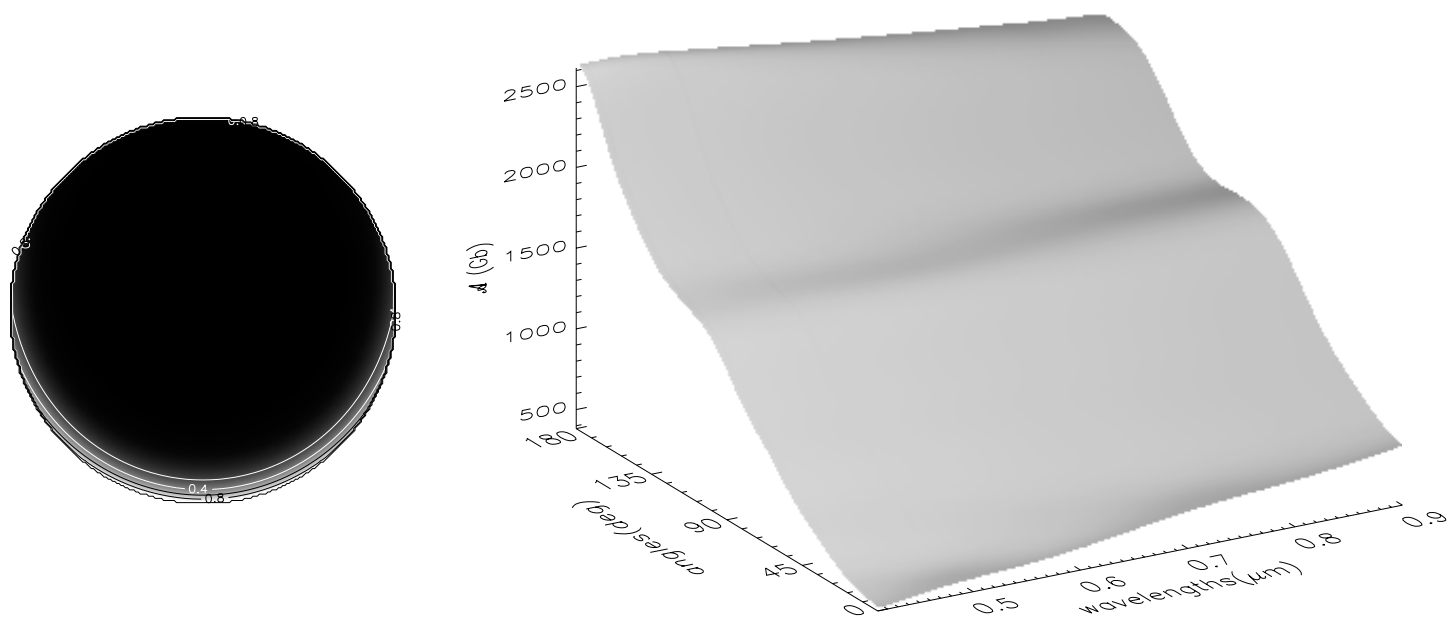

Fig. 11. Sample or2: incident wavelength $0.411 \mu \mathrm{m}$, sphere radius $0.95 \mu \mathrm{m}(x \sim 14.5)$.

Indeed, in Mulas et al. (2004) we consider two standard dust particle size distributions of the kind first proposed by Mathis et al. (1977), in order to infer the impact of the present model on the actual astrophysical problem of the Extended Red Emission.

In this case, $\frac{\mathrm{d} \mathcal{P}_{\mathrm{pl}}}{\mathrm{d} \Omega \mathrm{d} \omega^{\prime}}$ will retain only the overall large scale features of $\mathcal{A}\left(\omega, \omega^{\prime}, a, \theta, \theta_{\mathrm{d}}\right)$, while smoothing out the smaller oscillatory interference details (see Mulas et al. 2004). The overall behaviour will be governed by and large by the complex refractive index of the material.

\section{Conclusions and forthcoming work}

In spite of the extreme simplifications in its present numerical application, this model unambiguously demonstrates that self-absorption and geometric effects must be taken into account when comparing laboratory PL data taken on macroscopic samples with the observed PL from small particles, since they can have a rather important effect. Some perhaps misleading conclusions drawn from a direct comparison neglecting these effects may have to be regarded in a new light. In this respect, we present a more thorough analysis of the implications of this model for the specific case of the Extended
Red Emission in a second paper (Mulas et al. 2004). We expect to soon obtain new laboratory measurements of the optical and PL properties of various IPHAC samples in collaboration with the research group of Laboratory Astrophysics at the Catania Astrophysical Observatory, to use them along with the present model for a meaningful, quantitative comparison with available ERE observations.

To reconcile the observed under-solar abundances of heavy elements and the observed extinction, the present, state-of-theart interstellar dust models represent dust particles as complex aggregates (core-mantle and/or fluffy, porous grains), very different from our oversimplified homogeneous spheres. This makes it clear that the present application of our physical model of PL must be but a first step towards more realistic interstellar dust grain PL models. The first, and easiest unrealistic assumption which needs to be relaxed is homogeneity, in order to assess the impact of a multi-layer core-mantle structure on the outgoing PL while retaining spherical symmetry. To drop the latter is a more ambitious project, which will need a more sophisticated, and computationally expensive, approach, such as the T-matrix method, recently used to study the extinction of fluffy and porous dust grains (Iatì et al. 2001; Saja et al. 2001, 2003). This method is a natural match to our model, the major obstacle to its implementation being the need of much larger 
computing power resources to first find the coefficients of the expansions in VSHs and then numerically integrate the PL over the contributing volume of the particle.

On a parallel track, we want to extend our model to compute physical quantities related to other Stokes parameters besides I (as defined, e.g., in Bohren \& Huffman 1998, Sect. 2.11.1), to be able to study any polarisation effects stemming from the inhomogeneous distribution of absorption and resulting PL within the dust grain.

Acknowledgements. G. Malloci acknowledges the financial support by INAF - Osservatorio Astronomico di Cagliari. The authors are thankful to Prof. C. V. M. Van der Mee for his help with generalised spherical functions theory, and Dr. C. Cecchi-Pestellini and Prof. F. Borghese for their suggestions and useful discussions.

\section{References}

Bohren, C. F., \& Huffman, D. R. 1998, Absorption and scattering of light by small particles (New York: John Wiley \& Sons Inc.)

Chew, H., Kerker, M., \& McNulty, P. J. 1976a, J. Opt. Soc. Am., 66, 440

Chew, H., McNulty, P. J., \& Kerker, M. 1976b, Phys. Rev. A, 13, 396 De Rooij, W. A., \& Van der Stap, C. C. A. 1984, A\&A, 131, 237

Duley, W. W. 2001, ApJ, 553, 575

Dusel, P. W., Kerker, M., \& Cooke, D. D. 1976, J. Opt. Soc. Am., 69, 55

Greenberg, J. M., \& Pirronello, V. 1991, Chemistry in space

Hill, S. C., Saleheen, I., Barnes, M. D., Whitten, W. B., \& Ramsey, J. M. 1996, Appl. Opt., 35, 6278
Hovenier, J., \& Van der Mee, C. 1983, A\&A, 128, 1

Iatì, M. A., Cecchi-Pestellini, C., Williams, D. A., et al. 2001, MNRAS, 322, 749

Jackson, J. D. 1998, Classical Electrodynamics, 3d ed. (New York: John Wiley \& Sons Inc)

Jena, P., Khanna, S. N., \& Rao, B. K. N. 1992, Physics and Chemistry of Finite Systems: From Clusters to Crystals, Proc. of the NATO Advanced Research Workshop, Richmond, VA, USA, October 8-12, 1991 (Dordrecht: Kluwer Academic Publishers)

Jenniskens, P. 1993, A\&A, 274, 653

Kerker, M. 1973, Appl. Opt., 12, 1378

Kerker, M., \& Druger, S. 1979, Appl. Opt., 18, 1172

Kerker, M., McNulty, P. J., Sculley, M., Chew, H., \& Cooke, D. 1978a, J. Opt. Soc. Am., 68, 1676

Kerker, M., McNulty, P. J., Sculley, M., Chew, H., \& Cooke, D. D. 1978b, J. Opt. Soc. Am., 68(12), 1686

Li, A., \& Greenberg, J. M. 1997, A\&A, 323, 566

Malloci, G. 2003, Ph.D. Thesis, Università degli Studi di Cagliari

Mathis, J. S., Rumpl, W., \& Nordsieck, K. H. 1977, ApJ, 217, 425

Mathis, J. S., \& Whiffen, G. 1989, ApJ, 341, 808

Mulas, G., Malloci, G., \& Benvenuti, P. 2004, A\&A, 420, 921

Pendleton, D. J., \& Hill, S. C. 1997, Appl. Opt., 36, 8729

Robertson, J. 1996, Phys. Rev. B, 53, 16303

Rusli, G., Amaratunga, A. J., \& Robertson, J. 1996, Phys. Rev. B, 53, 16306

Saija, R., Iatì, M. A., Giusto, A., et al. 2003, MNRAS, 341, 1239

Saja, R., Iatí, M. A., Borghese, F., et al. 2001, ApJ, 559, 993

Seahra, S. S., \& Duley, W. W. 1999, ApJ, 529, 719

Smith, T. L., \& Witt, A. N. 2002, ApJ, 565, 304

Videen, G., Bickel, W. S., \& Boyer, J. M. 1991, Phys. Rev. A., 44, 1358

Wang, D., Kerker, M., \& Chew, W. 1980, Appl. Opt., 19, 2315 
G. Malloci et al.: Modelling photoluminescence from small particles. I., Online Material p 1

\section{Online Material}




\section{Appendix A: General formalism and basic concepts}

This appendix provides the basic concepts of the theoretical background of this work as well as the derivation of the main relations used in the application of our model of PL by small particles. We will discuss separately the two mechanisms of excitation and subsequent emission. The distribution of the absorbed energy inside the particle is presented in Sect. (A.1), while the treatment of the PL emission is given in Sect. (A.2). Section (A.3) provides the derivation of $p_{\text {loc }}$ for the case implemented in the present work. We followed the same units and notations adopted in Bohren \& Huffman's book (1998), henceforth B\&H. Details of the reductions to obtain the formulae here presented are given in Malloci (2003).

\section{A.1. Absorption}

We consider a spherical, isotropic and homogeneous dust particle of radius $a$. As depicted in Fig. 1, we choose the centre of the particle as the origin of a rectangular coordinate system $(x, y, z)$. We are interested in the distribution of the absorbed energy inside the particle under the hypothesis of incident natural light. Unpolarised light is described as the incoherent superposition of the two mutually orthogonal plane waves at frequency $\omega$, both of which are expanded in an infinite sum of vector spherical harmonics (henceforth VSHs). This expansion also dictates the form of the scattered and internal fields $(\mathrm{B} \& \mathrm{H})$.

Assuming the magnetic permeability of the particle to be the same as that of the surrounding medium, the resulting expression for the contribution of the volume element $\mathrm{d} V$ to $C_{\text {abs }}$ is given by:

$$
\begin{aligned}
\frac{\mathrm{d} C_{\mathrm{abs}}}{\mathrm{d} V}= & \frac{k \mathfrak{R}\left(m_{1}\right) \mathfrak{J}\left(m_{1}\right)}{\left|\rho_{0}\right|^{2}} \sum_{n, m=1}^{\infty} \frac{i^{n}(2 n+1)}{n(n+1)} \frac{(-i)^{m}(2 m+1)}{m(m+1)}\left[\left(c_{n} c_{m}^{*} \psi_{n} \psi_{m}^{*}+d_{n} d_{m}^{*} \psi_{n}^{\prime} \psi_{m}^{\prime}{ }^{*}\right)\left(\pi_{n} \pi_{m}+\tau_{n} \tau_{m}\right)\right. \\
& \left.+i\left(c_{n} d_{m}^{*} \psi_{n} \psi_{m}^{\prime *}-d_{n} c_{m}^{*} \psi_{n}^{\prime} \psi_{m}^{*}\right)\left(\pi_{n} \tau_{m}+\tau_{n} \pi_{m}\right)+n(n+1) m(m+1) \frac{\mathrm{d}_{n} d_{m}^{*} \psi_{n} \psi_{m}^{*}}{\left|\rho_{0}\right|^{2}} P_{n}^{1} P_{m}^{1}\right]
\end{aligned}
$$

where $m_{1}=k_{1} / k=N_{1} / N$ is the relative refractive index, $k_{1}=\omega N_{1} / c, k=\omega N / c$ and $N_{1}, N$ being the wave numbers and the refractive indices of particle and medium, respectively. $n$ and $m$ are positive integers and the prime, as usual, denotes differentiation with respect to the argument of the function, while the asterisk represents complex conjugation. The argument of the Riccati-Bessel functions $\psi_{n}$ is the dimensionless variable $\rho_{0}=k_{1} r_{0}=m_{1} k r_{0}$. The angular functions $\pi_{n}(\cos \theta)$ and $\tau_{n}(\cos \theta)$ are expressed in terms of the associated Legendre functions of the first kind $P_{n}^{1}$. The expansion coefficients $c_{n}$ and $d_{n}$ are completely determined by the size parameter $x$, related to the particle radius $a$ by $x=k a=\omega N a / c(\mathrm{~B} \& \mathrm{H})$.

Equation (A.1) is independent of the azimuthal angle $\phi$, as expected on physical grounds for unpolarised incident light. The integration over the volume $\mathcal{V}$ of the sphere leads to an expression of $C_{\text {abs }}$ in terms of $c_{n}$ and $d_{n}$ :

$C_{\mathrm{abs}}=\frac{2 \pi}{k^{2}\left|m_{1}\right|^{2}} \sum_{n=1}^{\infty}(2 n+1) \mathfrak{R}\left\{i \psi_{n}^{\prime}(y) \psi_{n}^{*}(y)\left[m_{1}\left|c_{n}\right|^{2}+m_{1}^{*}\left|d_{n}\right|^{2}\right]\right\}, \quad y=m_{1} x$,

which provides the same value for $C_{\mathrm{abs}}$ as obtained by the standard Lorenz-Mie theory $C_{\mathrm{ext}}-C_{\mathrm{sca}}(\mathrm{B} \& \mathrm{H})$.

\section{A.2. Emission}

We consider a dipole moment localised at position $\boldsymbol{r}_{\mathbf{0}}$ inside the sphere and oscillating at frequency $\omega^{\prime}$ :

$\boldsymbol{p}=\boldsymbol{p}_{0} \mathrm{e}^{-i \omega^{\prime} t}=\left\|\boldsymbol{p}_{0}\right\|\left(\sin \theta_{p} \cos \phi_{p} \hat{\boldsymbol{e}}_{x}+\sin \theta_{p} \sin \phi_{p} \hat{\boldsymbol{e}}_{y}+\cos \theta_{p} \hat{\boldsymbol{e}}_{z}\right) \mathrm{e}^{-i \omega^{\prime} t}=\left\|\boldsymbol{p}_{0}\right\|\left(\alpha \hat{\boldsymbol{e}}_{x}+\beta \hat{\boldsymbol{e}}_{y}+\gamma \hat{\boldsymbol{e}}_{z}\right) \mathrm{e}^{-i \omega^{\prime} t}$,

where we introduced the Euler angles $\theta_{p} \in[0, \pi]$ and $\phi_{p} \in[0,2 \pi]$ specifying the arbitrary orientation of the dipole. With no loss of generality, we will restrict the following derivation to $\boldsymbol{r}_{0}$ belonging to the positive $z$ axis, since any other position can be subsequently obtained with a simple rotation of the reference frame. Suppressing the time dependence $\mathrm{e}^{-i \omega^{\prime} t}$, the electromagnetic dipole fields occurring in Eq. (17) are given by:

$$
\begin{aligned}
& \boldsymbol{E}_{\mathrm{d}}(\boldsymbol{r})=\frac{1}{4 \pi \varepsilon_{1}} \frac{\mathrm{e}^{i k_{1} R}}{R}\left\{\left(\frac{3}{R^{2}}-\frac{3 i k_{1}}{R}-k_{1}^{2}\right) \frac{\boldsymbol{R}\left[\boldsymbol{R} \cdot \boldsymbol{p}_{0}\right]}{R^{2}}+\left(k_{1}^{2}+\frac{i k_{1}}{R}-\frac{1}{R^{2}}\right) \boldsymbol{p}_{0}\right\} \\
& \boldsymbol{H}_{\mathrm{d}}(\boldsymbol{r})=\frac{-i \omega^{\prime}}{4 \pi}\left\{\frac{\mathrm{e}^{i k_{1} R}}{R^{3}}\left(i k_{1} R-1\right)\left[\boldsymbol{r} \times \boldsymbol{p}_{0}\right]\right\},
\end{aligned}
$$

where $\boldsymbol{R}=\boldsymbol{r}-\boldsymbol{r}_{\mathbf{0}}, R=|\boldsymbol{R}|$ and $k_{1}$ and $\varepsilon_{1}$ are, respectively, the wave number and the permittivity of the medium composing the particle. We use the same notation as Jackson (1998), modified so that Eqs. (A.3) and (A.4) are valid for arbitrary dielectric media. To exploit the symmetry of the problem, it is advantageous to express the electromagnetic field as a series in vector spherical harmonics (VSHs). 
After a lengthy, although straightforward, procedure, the expansion of $\boldsymbol{E}_{\mathrm{d}}$ and $\boldsymbol{H}_{\mathrm{d}}$ in VSHs results in:

$$
\begin{aligned}
& \boldsymbol{E}_{\mathrm{d}}=\sum_{n=1}^{\infty} E_{n}\left\{\frac{\psi_{n}^{\prime}\left(\rho_{0}^{\prime}\right)}{\rho_{0}^{\prime}}\left[\alpha \boldsymbol{N}_{e 1 n}^{(3)}+\beta \boldsymbol{N}_{o 1 n}^{(3)}\right]+\frac{\psi_{n}\left(\rho_{0}^{\prime}\right)}{\rho_{0}^{\prime}}\left[\alpha \boldsymbol{M}_{o 1 n}^{(3)}-\beta \boldsymbol{M}_{e 1 n}^{(3)}\right]+n(n+1) \frac{\psi_{n}\left(\rho_{0}^{\prime}\right)}{\rho_{0}^{\prime 2}} \gamma \boldsymbol{N}_{e 0 n}^{(3)}\right\}, \\
& \boldsymbol{H}_{\mathrm{d}}=\sum_{n=1}^{\infty} H_{n}\left\{\frac{\psi_{n}^{\prime}\left(\rho_{0}^{\prime}\right)}{\rho_{0}^{\prime}}\left[\alpha \boldsymbol{M}_{e 1 n}^{(3)}+\beta \boldsymbol{M}_{o 1 n}^{(3)}\right]+\frac{\psi_{n}\left(\rho_{0}^{\prime}\right)}{\rho_{0}^{\prime}}\left[\alpha \boldsymbol{N}_{o 1 n}^{(3)}-\beta \boldsymbol{N}_{e 1 n}^{(3)}\right]+n(n+1) \frac{\psi_{n}\left(\rho_{0}^{\prime}\right)}{\rho_{0}^{\prime 2}} \gamma \boldsymbol{M}_{e 0 n}^{(3)}\right\},
\end{aligned}
$$

where $\rho_{0}^{\prime}=k_{1}^{\prime} r_{0}=m_{1} k^{\prime} r_{0}$ with $r_{0}=\left|\boldsymbol{r}_{0}\right|$ and we further defined:

$$
E_{n}=\frac{i \omega^{\prime 3} N_{1} \mu_{1}\left\|\boldsymbol{p}_{0}\right\|}{4 \pi c} \frac{2 n+1}{n(n+1)}, \quad H_{n}=\frac{\omega^{\prime 3}\left\|\boldsymbol{p}_{0}\right\| \varepsilon_{1} \mu_{1}}{4 \pi} \frac{2 n+1}{n(n+1)}, \quad E_{n}=i \sqrt{\frac{\mu_{1}}{\varepsilon_{1}}} H_{n} .
$$

Equations (A.5) and (A.6) are valid only for the radial domain $r_{0} \leq r \leq a$; this is sufficient for us, since we will need to evaluate the fields at the surface of the sphere in order to impose the boundary conditions. The electromagnetic fields are expected to show a singularity in the position where the dipole is located, i.e. $r=r_{0}$ and $\theta=0$; this singularity does not emerge from any single term in the expansion, but instead it is the series that is divergent in this case.

The expansion above, along with the orthogonality of the VSHs, the boundary conditions and the far field asymptotic behaviour expected, set the form of the complete solution, i.e. the expansion of both the scattered field $\left(\boldsymbol{E}_{\mathrm{s}}, \boldsymbol{H}_{\mathrm{s}}\right)$ and the outgoing field $\left(\boldsymbol{E}_{2}, \boldsymbol{H}_{2}\right)$. On physical grounds, the finiteness of the fields at the origin requires only the well behaved spherical Bessel functions of the first kind $j_{n}\left(k_{1} r\right)$ to be present in the expansion inside the sphere, whereas the spherical Bessel functions of the second kind $y_{n}\left(k_{1} r\right)$, which diverge for $r=0$, do not occur. The asymptotic behaviour of the spherical Hankel functions (B\&H) leads to the choice of $h_{n}^{(1)}(k r)=j_{n}(k r)+i y_{n}(k r)$ in the region outside the sphere, since the electromagnetic field leaving the particle must be an outgoing wave. Thus, appending as usual the superscript (1) which specifies that $j_{n}$ is the function containing the radial dependence in the VSHs:

$$
\begin{aligned}
& \boldsymbol{E}_{\mathrm{s}}=\sum_{n=1}^{\infty} E_{n}\left\{v_{n} \frac{\psi_{n}^{\prime}\left(\rho_{0}^{\prime}\right)}{\rho_{0}^{\prime}}\left[\alpha \boldsymbol{N}_{e 1 n}^{(1)}+\beta \boldsymbol{N}_{o 1 n}^{(1)}\right]+w_{n} \frac{\psi_{n}\left(\rho_{0}^{\prime}\right)}{\rho_{0}^{\prime}}\left[\alpha \boldsymbol{M}_{o 1 n}^{(1)}-\beta \boldsymbol{M}_{e 1 n}^{(1)}\right]+n(n+1) v_{n} \frac{\psi_{n}\left(\rho_{0}^{\prime}\right)}{\rho_{0}^{\prime 2}} \gamma \boldsymbol{N}_{e 0 n}^{(1)}\right\}, \\
& \boldsymbol{H}_{\mathrm{s}}=\sum_{n=1}^{\infty} H_{n}\left\{v_{n} \frac{\psi_{n}^{\prime}\left(\rho_{0}^{\prime}\right)}{\rho_{0}^{\prime}}\left[\alpha \boldsymbol{M}_{e 1 n}^{(1)}+\beta \boldsymbol{M}_{o 1 n}^{(1)}\right]+w_{n} \frac{\psi_{n}\left(\rho_{0}^{\prime}\right)}{\rho_{0}^{\prime}}\left[\alpha \boldsymbol{N}_{o 1 n}^{(1)}-\beta \boldsymbol{N}_{e 1 n}^{(1)}\right]+n(n+1) v_{n} \frac{\psi_{n}\left(\rho_{0}^{\prime}\right)}{\rho_{0}^{\prime 2}} \gamma \boldsymbol{M}_{e 0 n}^{(1)}\right\},
\end{aligned}
$$

where $v_{n}$ and $w_{n}$ are the coefficients to be determined, and $H_{n}$ and $E_{n}$ are the same as in Eq. (A.7). Likewise, introducing $a_{n}$ and $b_{n}$, another pair of coefficients to be determined, and appending the superscript (3) to VSHs whose radial dependence is expressed by $h_{n}^{(1)}$, the fields outside the particle are

$$
\begin{aligned}
& \boldsymbol{E}_{2}=\sum_{n=1}^{\infty} \widetilde{E}_{n}\left\{a_{n} \frac{\psi_{n}^{\prime}\left(\rho_{0}\right)}{\rho_{0}}\left[\alpha \boldsymbol{N}_{\mathrm{e} 1 \mathrm{n}}^{(3)}+\beta \boldsymbol{N}_{o 1 n}^{(3)}\right]+b_{n} \frac{\psi_{n}\left(\rho_{0}\right)}{\rho_{0}}\left[\alpha \boldsymbol{M}_{\mathrm{o} 1 \mathrm{n}}^{(3)}-\beta \boldsymbol{M}_{\mathrm{e} 1 \mathrm{n}}^{(3)}\right]+n(n+1) a_{n} \frac{\psi_{n}\left(\rho_{0}\right)}{\rho_{0}^{2}} \gamma \boldsymbol{N}_{\mathrm{e} 0 \mathrm{n}}^{(3)}\right\}, \\
& \boldsymbol{H}_{2}=\sum_{n=1}^{\infty} \widetilde{H}_{n}\left\{a_{n} \frac{\psi_{n}^{\prime}\left(\rho_{0}\right)}{\rho_{0}}\left[\alpha \boldsymbol{M}_{\mathrm{e} 1 \mathrm{n}}^{(3)}+\beta \boldsymbol{M}_{o 1 n}^{(3)}\right]+b_{n} \frac{\psi_{n}\left(\rho_{0}\right)}{\rho_{0}}\left[\alpha \boldsymbol{N}_{\mathrm{o} 1 \mathrm{n}}^{(3)}-\beta \boldsymbol{N}_{\mathrm{e} 1 \mathrm{n}}^{(3)}\right]+n(n+1) a_{n} \frac{\psi_{n}\left(\rho_{0}\right)}{\rho_{0}^{2}} \gamma \boldsymbol{M}_{\mathrm{e} 0 \mathrm{n}}^{(3)}\right\},
\end{aligned}
$$

where $\widetilde{E}_{n}$ and $\widetilde{H}_{n}$ are the expressions corresponding to (A.7) for the region outside the particle:

$\widetilde{E}_{n}=\frac{i \omega^{\prime 3} N \mu\left\|p_{0}\right\|}{4 \pi c} \frac{2 n+1}{n(n+1)}, \quad \widetilde{H}_{n}=\frac{\omega^{\prime 3}\left\|p_{0}\right\| \varepsilon \mu}{4 \pi} \frac{2 n+1}{n(n+1)} \quad \widetilde{E}_{n}=i \sqrt{\frac{\mu}{\varepsilon}} \widetilde{H}_{n}$.

The four unknown coefficients $a_{n}, b_{n}, v_{n}$ and $w_{n}$ are obtained imposing the usual boundary conditions across the sphere surface. After some manipulation, making use of the orthogonality of VSHs one obtains:

$a_{n}=\frac{\mu_{1} m_{1}\left[\xi_{n}\left(y^{\prime}\right) \psi_{n}^{\prime}\left(y^{\prime}\right)-\xi_{n}^{\prime}\left(y^{\prime}\right) \psi_{n}\left(y^{\prime}\right)\right]}{\mu_{1} \xi_{n}\left(x^{\prime}\right) \psi_{n}^{\prime}\left(y^{\prime}\right)-\mu m_{1} \xi_{n}^{\prime}\left(x^{\prime}\right) \psi_{n}\left(y^{\prime}\right)}, \quad b_{n}=\frac{\mu_{1} m_{1}\left[\xi_{n}^{\prime}\left(y^{\prime}\right) \psi_{n}\left(y^{\prime}\right)-\xi_{n}\left(y^{\prime}\right) \psi_{n}^{\prime}\left(y^{\prime}\right)\right]}{\mu_{1} \xi_{n}^{\prime}\left(x^{\prime}\right) \psi_{n}\left(y^{\prime}\right)-\mu m_{1} \xi_{n}\left(x^{\prime}\right) \psi_{n}^{\prime}\left(y^{\prime}\right)}$,

$v_{n}=\frac{\mu m_{1} \xi_{n}\left(y^{\prime}\right) \xi_{n}^{\prime}\left(x^{\prime}\right)-\mu_{1} \xi_{n}^{\prime}\left(y^{\prime}\right) \xi_{n}\left(x^{\prime}\right)}{\mu_{1} \xi_{n}\left(x^{\prime}\right) \psi_{n}^{\prime}\left(y^{\prime}\right)-\mu m_{1} \xi_{n}^{\prime}\left(x^{\prime}\right) \psi_{n}\left(y^{\prime}\right)}, \quad w_{n}=\frac{\mu m_{1} \xi_{n}^{\prime}\left(y^{\prime}\right) \xi_{n}\left(x^{\prime}\right)-\mu_{1} \xi_{n}\left(y^{\prime}\right) \xi_{n}^{\prime}\left(x^{\prime}\right)}{\mu_{1} \xi_{n}^{\prime}\left(x^{\prime}\right) \psi_{n}\left(y^{\prime}\right)-\mu m_{1} \xi_{n}\left(x^{\prime}\right) \psi_{n}^{\prime}\left(y^{\prime}\right)}$,

with $y^{\prime}=m_{1} x^{\prime}=m_{1} k^{\prime} a$. The denominators of $v_{n}$ and $a_{n}$ are identical, as are those of $w_{n}$ and $b_{n}$. As in the previous section, making the assumption $\mu_{1}=\mu$ we have:

$a_{n}=\frac{m_{1}\left[\xi_{n}\left(y^{\prime}\right) \psi_{n}^{\prime}\left(y^{\prime}\right)-\xi_{n}^{\prime}\left(y^{\prime}\right) \psi_{n}\left(y^{\prime}\right)\right]}{\xi_{n}\left(x^{\prime}\right) \psi_{n}^{\prime}\left(y^{\prime}\right)-m_{1} \xi_{n}^{\prime}\left(x^{\prime}\right) \psi_{n}\left(y^{\prime}\right)}, \quad b_{n}=\frac{m_{1}\left[\xi_{n}^{\prime}\left(y^{\prime}\right) \psi_{n}\left(y^{\prime}\right)-\xi_{n}\left(y^{\prime}\right) \psi_{n}^{\prime}\left(y^{\prime}\right)\right]}{\xi_{n}^{\prime}\left(x^{\prime}\right) \psi_{n}\left(y^{\prime}\right)-m_{1} \xi_{n}\left(x^{\prime}\right) \psi_{n}^{\prime}\left(y^{\prime}\right)}$, 
$v_{n}=\frac{m_{1} \xi_{n}\left(y^{\prime}\right) \xi_{n}^{\prime}\left(x^{\prime}\right)-\xi_{n}^{\prime}\left(y^{\prime}\right) \xi_{n}\left(x^{\prime}\right)}{\xi_{n}\left(x^{\prime}\right) \psi_{n}^{\prime}\left(y^{\prime}\right)-m_{1} \xi_{n}^{\prime}\left(x^{\prime}\right) \psi_{n}\left(y^{\prime}\right)}, \quad w_{n}=\frac{m_{1} \xi_{n}^{\prime}\left(y^{\prime}\right) \xi_{n}\left(x^{\prime}\right)-\xi_{n}\left(y^{\prime}\right) \xi_{n}^{\prime}\left(x^{\prime}\right)}{\xi_{n}^{\prime}\left(x^{\prime}\right) \psi_{n}\left(y^{\prime}\right)-m_{1} \xi_{n}\left(x^{\prime}\right) \psi_{n}^{\prime}\left(y^{\prime}\right)}$

When the particle and the surrounding medium have the same optical properties, the transmitted fields must coincide with the dipole fields, while the scattered fields must vanish. Indeed, in the $m_{1} \rightarrow 1$ limit $a_{n}$ an $b_{n}$ approach unity, while $v_{n}$ and $w_{n}$ vanish.

We are eventually interested in the total power irradiated by the particle at very large distances from it, into a small solid angle about a given direction. In the far-field region the radial components $E_{r}$ and $H_{r}$ of the external fields become negligible with respect to the angular ones, which are related by:

$H_{\phi}=\sqrt{\frac{\varepsilon}{\mu}} E_{\theta}, \quad H_{\theta}=-\sqrt{\frac{\varepsilon}{\mu}} E_{\phi}$.

This follows from the asymptotic behaviour of the Riccati-Bessel function $\xi_{n}$ and its derivative (B\&H). Thus, the time-averaged Poynting vector in the far- field is given by:

$\langle\boldsymbol{S}\rangle\left(\omega^{\prime}, \boldsymbol{r}, \boldsymbol{r}_{0}, \Omega_{p}\right)=\frac{1}{2} \mathfrak{R}\left\{\boldsymbol{E}_{2}^{\mathrm{ff}} \times \boldsymbol{H}_{2}^{\mathrm{ff}}\right\}=\frac{1}{2} \mathfrak{R}\left\{E_{\theta} H_{\phi}^{*}-E_{\phi} H_{\theta}^{*}\right\} \hat{\boldsymbol{e}}_{r}=\frac{1}{2} \sqrt{\frac{\varepsilon}{\mu}}\left\{\left|E_{\theta}\right|^{2}+\left|E_{\phi}\right|^{2}\right\} \hat{\boldsymbol{e}}_{r}$.

The expression of $E_{\theta}$ and $E_{\phi}$ is given in Malloci (2003).

So far we have been considering a single, discrete dipole, oscillating at a given frequency. We can move to a continuous distribution of dipoles with the obvious substitutions

$\boldsymbol{p}_{0} \rightarrow \frac{\mathrm{d} p_{0}}{\mathrm{~d} \omega^{\prime} \mathrm{d} V_{0} \mathrm{~d} \Omega_{p}}$

and

$\langle\boldsymbol{S}\rangle \rightarrow \frac{\mathrm{d}\langle\boldsymbol{S}\rangle}{\mathrm{d} \omega^{\prime} \mathrm{d} V_{0} \mathrm{~d} \Omega_{p}}$

The incoherent sum over all possible orientations of the dipoles yields

$\frac{\mathrm{d}\langle\boldsymbol{S}\rangle}{\mathrm{d} \omega^{\prime} \mathrm{d} V_{0}}=\int_{4 \pi} \frac{\mathrm{d}\langle\boldsymbol{S}\rangle}{\mathrm{d} \omega^{\prime} \mathrm{d} V_{0} \mathrm{~d} \Omega_{p}} \mathrm{~d} \Omega_{p}=\int_{0}^{2 \pi} \int_{0}^{\pi} \frac{\mathrm{d}\langle\boldsymbol{S}\rangle}{\mathrm{d} \omega^{\prime} \mathrm{d} V_{0} \mathrm{~d} \Omega_{p}} \sin \theta_{p} \mathrm{~d} \theta_{p} \mathrm{~d} \phi_{p}$,

which can be evaluated observing that:

$\int_{4 \pi} \alpha \beta \mathrm{d} \Omega_{p}=\int_{4 \pi} \alpha \gamma \mathrm{d} \Omega_{p}=\int_{4 \pi} \beta \gamma \mathrm{d} \Omega_{p}=0, \quad$ and $\quad \int_{4 \pi} \alpha^{2} \mathrm{~d} \Omega_{p}=\int_{4 \pi} \beta^{2} \mathrm{~d} \Omega_{p}=\int_{4 \pi} \gamma^{2} \mathrm{~d} \Omega_{p}=\frac{4 \pi}{3}$.

The total power radiated per unit solid angle at frequency $\omega^{\prime}$ by this isotropic collection of dipoles centred in $\boldsymbol{r}_{0}$ is hence given by:

$\frac{\mathrm{d} \mathcal{P}_{\mathrm{pl}}}{\mathrm{d} \Omega \mathrm{d} \omega^{\prime} \mathrm{d} V_{0}}=\left\|\frac{\mathrm{d}\langle\boldsymbol{S}\rangle}{\mathrm{d} \omega^{\prime} \mathrm{d} V_{0}}\right\| r^{2}$

If the medium surrounding the particle is non-absorbing (not necessarily the vacuum) this quantity at large distances must be independent of $r$; it only depends on the position $\boldsymbol{r}_{0}$ of the dipole inside the grain and the direction $\mathrm{d} \Omega$ at which emission is observed.

With the help of Eq. (A.12) defining $\widetilde{E}_{n}$, we obtain, after some manipulation, Eq. (18) of Sect. 2.2:

$\frac{\mathrm{d} \mathcal{P}_{\mathrm{pl}}}{\mathrm{d} \Omega \mathrm{d} \omega^{\prime} \mathrm{d} V_{0}}=4 \pi \frac{\mathrm{d} \mathcal{P}_{\mathrm{dip}}}{\mathrm{d} \omega^{\prime} \mathrm{d} V_{0} \mathrm{~d} \Omega_{p}} \frac{\mathrm{d} \mathcal{F}}{\mathrm{d} \Omega}$.

The expression of $\frac{\mathrm{d} \mathcal{F}}{\mathrm{d} \Omega}$ for a homogeneous, isotropic sphere is given by:

$$
\begin{aligned}
\frac{\mathrm{d} \mathcal{F}}{\mathrm{d} \Omega}= & \frac{1}{8 \pi \Re\left[m_{1}\left(\omega^{\prime}\right)\right]\left|\rho_{0}^{\prime}\right|^{2}} \sum_{p, q=1}^{\infty} \frac{(-i)^{p}(2 p+1)}{p(p+1)} \frac{i^{q}(2 q+1)}{q(q+1)}\left[\left(b_{p} b_{q}^{*} \psi_{p} \psi_{q}^{*}+a_{p} a_{q}^{*} \psi_{p}^{\prime} \psi_{q}^{\prime *}\right)\left(\pi_{p} \pi_{q}+\tau_{p} \tau_{q}\right)\right. \\
& \left.-i\left(b_{p} a_{q} * \psi_{p} \psi_{q}^{\prime *}-a_{p} b_{q}^{*} \psi_{p}^{\prime} \psi_{q}^{*}\right)\left(\pi_{p} \tau_{q}+\tau_{p} \pi_{q}\right)+p(p+1) q(q+1) \frac{a_{p} a_{q}^{*} \psi_{p} \psi_{q}^{*}}{\left|\rho_{0}^{\prime}\right|^{2}} P_{p}^{1} P_{q}^{1}\right],
\end{aligned}
$$

where $a_{j}$ and $b_{j}$ are given by Eq. (A.13). If $m_{1}\left(\omega^{\prime}\right) \equiv 1$ it is easily found that

$\int_{4 \pi} \mathrm{d} \Omega \frac{\mathrm{d} \mathcal{F}}{\mathrm{d} \Omega}=1$ 


\section{G. Malloci et al.: Modelling photoluminescence from small particles. I., Online Material p 5}

Equation (A.18) is obviously $\phi$-independent, since we restricted $\boldsymbol{r}_{0}$ to be along the $z$-axis and summed over all possible orientations. The same Eq. (A.18) turns out to also provide the expression of $\frac{\mathrm{d} \mathcal{F}}{\mathrm{d} V}$ for an arbitrary $\boldsymbol{r}_{0}$, with just the simple substitution $\cos \theta \rightarrow \cos \Theta=\sin \theta \sin \theta_{0} \cos \left(\phi-\phi_{0}\right)+\cos \theta \cos \theta_{0}$.

in the arguments of the angular functions $\pi_{j}, \tau_{j}$ and $P_{j}^{1}$ into Eq. (A.18).

We thus obtained all of the ingredients occurring in Eq. (25) in the specific case of a homogeneous, isotropic sphere. The angular integrations can be evaluated analytically expanding the angular functions contained in $\frac{\mathrm{d} C_{\mathrm{abs}}}{\mathrm{d} V}$ and $\frac{\mathrm{d} \mathcal{F}}{\mathrm{d} \Omega}$ as linear combinations of generalised spherical functions (GSFs) $P_{m, n}^{l}$ (Hovenier \& Van der Mee 1983), which yields

$\frac{\mathrm{d} C_{\mathrm{abs}}}{\mathrm{d} V}=\frac{k \mathfrak{R}\left[m_{1}(\omega)\right] \mathfrak{J}\left[m_{1}(\omega)\right]}{2}\left\{\sum_{n=1}^{\infty} \sum_{r=0}^{2 n} \mathcal{B}_{n n r}\left(\rho_{0}\right) P_{r}\left(\cos \theta_{0}\right)+2 \sum_{n=1}^{\infty} \sum_{m>n}^{\infty} \sum_{r=m-n}^{m+n} \mathcal{B}_{m n r}\left(\rho_{0}\right) P_{r}\left(\cos \theta_{0}\right)\right\}$

and

$\frac{\mathrm{d} \mathcal{F}}{\mathrm{d} \Omega}=\frac{1}{16 \pi \mathfrak{R}\left[m_{1}\left(\omega^{\prime}\right)\right]}\left\{\sum_{p=1}^{\infty} \sum_{s=0}^{2 p} \mathcal{D}_{p p s}\left(\rho_{0}^{\prime}\right) P_{s}(\cos \Theta)+2 \sum_{p=1}^{\infty} \sum_{q>p}^{\infty} \sum_{s=q-p}^{q+p} \mathcal{D}_{q p s}\left(\rho_{0}^{\prime}\right) P_{s}(\cos \Theta)\right\}$,

where the coefficients of the expansions are:

$$
\begin{aligned}
\mathcal{B}_{m n r}= & \frac{(2 r+1)}{\left|\rho_{0}\right|^{2}}\left\{\mathfrak{R}\left[f_{n} f_{m}^{*}\left(c_{n} \psi_{n} c_{m}^{*} \psi_{m}^{*}+d_{n} \psi_{n}^{\prime} d_{m}^{*} \psi_{m}^{\prime *}\right)\right]\left(A_{r, n}^{m}\right)^{2}\left[1+(-1)^{m+n+r}\right]\right. \\
& \left.-\mathfrak{J}\left[f_{n} f_{m}^{*}\left(c_{n} \psi_{n} d_{m}^{*} \psi_{m}^{\prime *}-d_{n} \psi_{n}^{\prime} c_{m}^{*} \psi_{m}^{*}\right)\right]\left(A_{r, n}^{m}\right)^{2}\left[1-(-1)^{m+n+r}\right]-2 \sqrt{g_{n} g_{m}} \frac{\mathfrak{R}\left(f_{n} f_{m}^{*} d_{n} \psi_{n} d_{m}^{*} \psi_{m}^{*}\right)}{\left|\rho_{0}\right|^{2}} A_{r, n}^{m} C_{r, n}^{m}\right\}, \\
\mathcal{D}_{q p s}= & \frac{(2 s+1)}{\left|\rho_{0}^{\prime}\right|^{2}}\left\{\mathfrak{R}\left[f_{p}^{*} f_{q}\left(b_{p} \psi_{p} b_{q}^{*} \psi_{q}^{*}+a_{p} \psi_{p}^{\prime} a_{q}^{*} \psi_{q}^{\prime *}\right)\right]\left(A_{s, p}^{q}\right)^{2}\left[1+(-1)^{q+p+s}\right]\right. \\
& \left.+\mathfrak{J}\left[f_{p}^{*} f_{q}\left(b_{p} \psi_{p} a_{q}^{*} \psi_{q}^{\prime *}-a_{p} \psi_{p}^{\prime} b_{q}^{*} \psi_{q}^{*}\right)\right]\left(A_{s, p}^{q}\right)^{2}\left[1-(-1)^{q+p+s}\right]-2 \sqrt{g_{p} g_{q}} \frac{\mathfrak{R}\left(f_{p}^{*} f_{q} a_{p} \psi_{p} a_{q}^{*} \psi_{q}^{*}\right)}{\left|\rho_{0}^{\prime}\right|^{2}} A_{s, p}^{q} C_{s, p}^{q}\right\},
\end{aligned}
$$

and the meaning of the newly introduced terms is:

$$
f_{j}=(i)^{j}(2 j+1), \quad g_{j}=j(j+1), \quad A_{l, n}^{m}=\left(\begin{array}{ccc}
m & n & r \\
-1 & 1 & 0
\end{array}\right), \quad C_{l, n}^{m}=\left(\begin{array}{ccc}
m & n & r \\
0 & 0 & 0
\end{array}\right),
$$

the last two terms being Wigner 3j-symbols, well known in the quantum theory of angular momentum (see e. g. De Rooij \& Van der Stap 1984). The infinite sums in the above equations can be truncated, for practical purposes, to a finite number of terms sufficient to make the truncation error negligible $(\mathrm{B} \& \mathrm{H})$. We will call $N$ and $P$ the number of terms required for the expansions in $\frac{\mathrm{d} C_{\mathrm{abs}}}{\mathrm{d} V}$ and $\frac{\mathrm{d} \mathcal{F}}{\mathrm{d} \Omega}$, respectively.

Substituting Eqs. (A.19) and (A.20) in Eq. (25), the angular integration can be evaluated making use of the properties of the GSFs yielding, after a hearty amount of algebra which we spare the reader:

$$
\frac{\mathrm{d} C_{p l}}{\mathrm{~d} \Omega \mathrm{d} \omega^{\prime}}=\frac{\omega^{\prime}}{\omega} p_{\mathrm{loc}}\left(\omega^{\prime}, \omega\right) \sum_{t=0}^{t^{*}} \gamma_{t}\left(a, \omega, \omega^{\prime}\right) P_{t}(\cos \theta)
$$

with

$\gamma_{t}=\frac{k \mathfrak{R}\left[m_{1}(\omega)\right] \mathfrak{J}\left[m_{1}(\omega)\right]}{8 \mathfrak{R}\left[m_{1}\left(\omega^{\prime}\right)\right]}(2 t+1) \int_{0}^{a} r_{0}^{2}\left(\sum_{n=n^{*}}^{N} \mathcal{B}_{n n t}+2 \sum_{n=1}^{N-1} \sum_{m=m^{*}}^{m^{* *}} \mathcal{B}_{m n t}\right)\left(\sum_{p=p^{*}}^{P} \mathcal{D}_{p p t}+2 \sum_{p=1}^{P-1} \sum_{q=q^{*}}^{q^{* *}} \mathcal{D}_{q p t}\right) \mathrm{d} r_{0}$,

$t^{*}=\min (2 N, 2 P), \quad n^{*}=\max \left(1, \frac{t+1}{2}\right), \quad p^{*}=\max \left(1, \frac{t+1}{2}\right), m^{*}=\max (n+1, t-n)$,

$m^{* *}=\min (t+n, N), \quad q^{*}=\max (p+1, t-p), \quad$ and $\quad q^{* *}=\max (t+p, P)$.

Details of the numerical methods used to evaluate each term of Eq. (A.21) are given in Malloci (2003). 


\section{A.3. The evaluation of $p_{\mathrm{loc}}$}

We need to practically evaluate Eq. (26), to obtain $p_{\text {loc }}\left(\omega, \omega^{\prime}\right)$ from laboratory measurements of the PL yield of a bulk sample in a specific experimental setup. We consider the experimental configuration of Fig. 2, in which a bulk sample is illuminated by a collimated laser beam of frequency $\omega$ travelling in the positive $z$ direction, and the PL emission at frequency $\omega^{\prime}$ is observed at angle $\theta_{\mathrm{d}}$ with respect to the exciting radiation. We may rewrite Eq. (26) multiplying both the numerator and the denominator by the irradiance $I(\omega)$ of the incident beam and the total power $\mathcal{P}_{\text {abs }}$ absorbed by the sample, to obtain:

$p_{\mathrm{loc}}\left(\omega, \omega^{\prime}\right)=\frac{\omega}{\omega^{\prime}} \cdot \frac{I(\omega) \mathcal{P}_{\mathrm{abs}}}{I(\omega) \mathcal{P}_{\mathrm{abs}}} \cdot \frac{\left(\frac{\mathrm{d} C_{\mathrm{pl}}}{\mathrm{d} \Omega \mathrm{d} \omega^{\prime}}\right)_{\mathrm{lab}}}{\left(\int_{\mathcal{V}} \frac{\mathrm{d} C_{\mathrm{abs}}}{\mathrm{d} V} \frac{\mathrm{d}}{\mathrm{d} \Omega} \mathrm{d} V\right)_{\mathrm{lab}}}=\frac{\omega}{\omega^{\prime}} \cdot \frac{\mathrm{d} \eta_{\mathrm{lab}}}{\mathrm{d} \Omega \mathrm{d} \omega^{\prime}} \cdot \frac{\mathcal{P}_{\mathrm{abs}}}{\left(\int_{\mathcal{V}} \frac{\mathrm{d} \mathcal{P}_{\mathrm{abs}}}{\mathrm{d} V} \frac{\mathrm{f} \mathcal{F}}{\mathrm{d} \Omega} \mathrm{d} V\right)_{\mathrm{lab}}}$,

where we used Eqs. (24) and (31) to introduce the experimental differential PL yield.

To be able to derive $p_{\text {loc }}\left(\omega, \omega^{\prime}\right)$ from the experimental measurement of $\frac{\mathrm{d} \eta_{\text {lab }}}{\mathrm{d} \Omega \mathrm{d} \omega^{\prime}}$ we must now evaluate the fraction

$$
\frac{1}{g\left(\omega, \omega^{\prime}\right)}=\frac{\mathcal{P}_{\mathrm{abs}}}{\left(\int_{\mathcal{V}} \frac{\mathrm{d} \mathcal{P}_{\mathrm{abs}}}{\mathrm{d} V} \frac{\mathrm{d} \mathcal{F}}{\mathrm{d} \Omega} \mathrm{d} V\right)_{\mathrm{lab}}}
$$

This must generally involve a detailed analysis of the propagation of the light impinging on the sample, its absorption and the propagation of the resulting PL up to the collecting optics of the detector, which in turn will require the expansion of the electromagnetic fields using a basis appropriate to the geometry of the sample and to its symmetry (i.e. usually plane waves). However, if the material considered is sufficiently absorbing, it is possible to reuse some of the results obtained in the previous sections for a sphere.

In a typical experimental configuration, the collimating optics will have a diameter of the order of $2 \mathrm{~cm}$, a focal length of the order of $15 \mathrm{~cm}$, and will illuminate a spot of less than $10 \mu \mathrm{m}$ on the surface of the sample, when focused. The parallel laser beam before focusing has a diameter of the order of $2 \mathrm{~mm}$. This beam penetrates into the sample for a depth of the order of a few times $1 / \alpha(\omega), \alpha(\omega)$ being the absorption coefficient at incident wavelength, which translates into less than $10 \mu \mathrm{m}$ for the organic refractory residues considered for the examples in this work.

Given its small aperture, the relatively large focal length and the short path it travels inside the sample, the collimated laser beam can be considered as a portion of a parallel beam, confined into a cylinder: the diameter of the beam will change by less than one part in $10^{4}$ due to its divergence before being completely absorbed. Hence, we represent this experimental configuration as a plane parallel slab in which a cylinder of diameter $\lesssim 10 \mu \mathrm{m}$ is illuminated by a normally incident portion of plane wave, which is exponentially attenuated as it travels inwards, so that only about $10 \mu \mathrm{m}$ of its length need to be considered. In the local PL limit, only this same cylinder will produce luminescence photons. It is clear that as long as only such a small portion of the sample is participating in the PL, we can consider this portion to be a part of a sphere, provided it is large enough for the curvature of the surface to be negligible over lengths of the order of the absorption length. In this geometry, the illuminated part of the sample (imagined as a sphere centred in the origin of the reference system and illuminated by a parallel beam along the $z$-axis) can be approximated by a small portion of a narrow right circular cone of aperture $2 \Delta \gamma$ centred around the beam axis and having its vertex in the centre of the sphere. This frustum of cone, for sphere radius much larger than the cylinder section, becomes undistinguishable from the actually illuminated cylinder we are considering. If the circular spot illuminated on the sample surface has area $\Delta S$, the aperture $2 \Delta \gamma$ of the frustum of cone will be given by the relation $\Delta S=\pi(\Delta \gamma a)^{2}$. We remark that, for the sizes, wavelengths and optical properties we are considering, a sphere of radius of the order of $1 \mathrm{~mm}$ is already quite large enough. Since the outgoing light is collected at distances of the order of $\sim 15 \mathrm{~cm}$, we can still quite safely consider it to be in the far field limit. The power absorbed by a unit volume within the illuminated cylinder (or frustum of cone, defined by $\left.\theta_{0} \in[\pi-\Delta \gamma, \pi]\right)$, can be thus written as

$$
\frac{\mathrm{d} \mathcal{P}_{\mathrm{abs}}}{\mathrm{d} V}=I(\omega) \mathcal{T}(\omega) \alpha(\omega) \mathrm{e}^{-\alpha(\omega)\left(a-r_{0}\right)}
$$

$a$ being the radius of our "large" sphere, $r_{0}$ the radial coordinate, $\alpha(\omega)$ the absorption coefficient at incident wavelength and $\mathcal{T}(\omega)$ the transmittance for normal incidence at a plane boundary. $\frac{\mathrm{d} \mathcal{P}_{\text {abs }}}{\mathrm{d} V}$ is assumed to be identically zero outside of the illuminated cylinder (which we will approximate by the frustum of cone in the integration). In essence, we just use geometric optics for the absorption, which is fully justified for the experimental conditions described above.

The integral at the denominator of the right hand side of Eq. (A.24) can thus be written as

$$
\left(\int_{\mathcal{V}} \frac{\mathrm{d} \mathcal{P}_{\mathrm{abs}}}{\mathrm{d} V} \frac{\mathrm{d} \mathcal{F}}{\mathrm{d} \Omega} \mathrm{d} V\right)_{\mathrm{lab}} \simeq I(\omega) \mathcal{T} \alpha(\omega) \Delta S \int_{0}^{a}\left(\frac{r_{0}}{a}\right)^{2} \mathrm{e}^{-\alpha(\omega)\left(a-r_{0}\right)} \frac{\mathrm{d} \mathcal{F}}{\mathrm{d} \Omega} \mathrm{d} r_{0}
$$


the approximate equality being due to approximating the illuminated cylinder (the integration domain) with the frustum of cone. The total power absorbed by the sample is

$\mathcal{P}_{\mathrm{abs}}=I(\omega) \mathcal{T}(\omega) \Delta S \simeq I(\omega) \mathcal{T}(\omega) \alpha(\omega) \Delta S \int_{0}^{a}\left(\frac{r_{0}}{a}\right)^{2} \mathrm{e}^{-\alpha(\omega)\left(a-r_{0}\right)} \mathrm{d} r_{0}$,

the approximate equality sign again due to the approximation of the cylinder with the frustum of cone. This equation can be used to quantify the accuracy of this approximation, since the integral on the right hand side can be evaluated analytically to yield

$\int_{0}^{a}\left(\frac{r_{0}}{a}\right)^{2} \mathrm{e}^{-\alpha(\omega)\left(a-r_{0}\right)} \mathrm{d} r_{0}=\frac{1}{\alpha}\left(1+\frac{-2 \alpha a+2-2 \mathrm{e}^{-\alpha a}}{(\alpha a)^{2}}\right)$,

which rapidly approaches its limiting value of $1 / \alpha$ for increasing $a$.

With the help of the previous equations, we can write

$g\left(\omega, \omega^{\prime}\right) \simeq \lim _{a \rightarrow \infty}\left(\frac{\int_{0}^{a} r_{0}^{2} \mathrm{e}^{-\alpha(\omega)\left(a-r_{0}\right)} \frac{\mathrm{d} \mathcal{F}}{\mathrm{d} \Omega} \mathrm{d} r_{0}}{\int_{0}^{a} r_{0}^{2} \mathrm{e}^{-\alpha(\omega)\left(a-r_{0}\right)} \mathrm{d} r_{0}}\right)$.

For every given couple of frequencies $\omega$ and $\omega^{\prime}$, we numerically evaluated the above equation for increasing sphere radii $a$, until convergence was reached with an accuracy of three significant figures. This was achieved, in all cases considered in the example application presented in this work, for $a \lesssim 100 \mu \mathrm{m}$. We remark that neither $I(\omega)$ nor $\Delta S$ are present in the above equation, as would be expected on physical grounds.

Substituting Eqs. (A.24) and (31) into Eq. (A.23) yields the desired expression for $p_{\text {loc }}\left(\omega, \omega^{\prime}\right)$ in terms of measurable and calculable quantities:

$p_{\text {loc }}\left(\omega, \omega^{\prime}\right)=\frac{1}{g\left(\omega, \omega^{\prime}\right)} \frac{\omega}{\omega^{\prime}} \frac{\mathrm{d} \eta_{\text {lab }}}{\mathrm{d} \Omega \mathrm{d} \omega^{\prime}}$,

where we use Eq. (A.28) to evaluate $g\left(\omega, \omega^{\prime}\right)$. 